\title{
Non-Linear Analysis of the NASA Super Pressure Balloons: Whole Flight Simulations
}

\author{
David Wakefield * and Adam Bown ${ }^{\dagger}$ \\ Tensys Dynamics Ltd., Bath BA1 5BG UK
}

\begin{abstract}
Tensys have a long-established background in the shape generation and load analysis of architectural stressed membrane structures. Founded upon their inTENS finite element analysis suite, these activities have broadened to encompass 'lighter than air' structures such as aerostats, airships, hybrid air vehicles and stratospheric balloons. Tensys have acted as consultants to the NASA Super Pressure Balloon (SPB) Program since 2004.

Previous papers have focussed upon the application of inTENS to the overall structural and stability analysis of pumpkin type balloons as used in the SPB Program. Particular emphasis has been placed upon the ability to study both stress and stability at all stages of a flight. As the program has developed, increasing modelling fidelity has been introduced as design refinement has moved emphasis from the overall shape to the performance of individual details. Examples include the introduction of a contact capability into inTENS to better represent the separate cap layer of film. Localised investigations have considered the consequence of local geometric anomalies introduced during fabrication and the effects of debonding of the PBO tendon within its sleeve.
\end{abstract}

Analysis to date has used a material model for the polyethylene shell film developed by Dr Rand of Winzen Engineering, supported by a program of fine resolution material tests at the Balloon Research and Development Laboratory (BRDL) at the GSFC Wallops Island Facility. Based upon work first reported by Schapery, this model has been incorporated into so-called 'snapshot' analyses within inTENS. For a given elapsed time point and temperature distribution, non-linear material properties are iteratively updated for the current stress state in each individual element until changes in those material properties are insignificant. This process has to be iterative as the film stresses and material properties are interdependent.

Attempts to derive a time-stepping incremental viscoelastic capability using the Schapery Rand model encountered problems when dealing with the varying temperatures associated with balloon deployment, pressurisation and diurnal behaviour. An alternative approach has been developed by Pellegrino et al at Caltech, again with support from the NASA Balloon Program Office. This is a large strain non-linear viscoelastic model that includes film out-ofplane mechanical and thermal effects. Working from a unified base, this model can be utilised for time-stepping analyses in both a modulus or compliance mode, or a combination of both.

This new capability is intended to enhance the current NASA balloon design process. This paper presents the implementation of the Caltech model into a specialist finite element suite, inTENS and its application to whole flight simulations.

* Director, Tensys Dynamics Ltd, 1 St Swithins Yard, Walcot Street, Bath BA1 5BG, UK

${ }^{\dagger}$ Director, Tensys Dynamics Ltd, 1 St Swithins Yard, Walcot Street, Bath BA1 5BG, UK

AIAA Associate Fellow 


\section{High Altitude Scientific Balloons: NASA Super Pressure Balloon Program}

\section{A. Background}

The NASA Super-Pressure Balloons (SPB, fig 1) are designed to carry scientific payloads of up to 3,500kg around the globe on flights of up to 100 days duration at altitudes of between 35,000 and $40,000 \mathrm{~m}$. Of 'pumpkin' form, and up to $120 \mathrm{~m}$ in diameter, these balloons are fabricated from typically 38 micron polyethylene film combined with PBO tendons spanning between the base and apex end fittings. The particular feature of the pressurized SPB when compared to its zero pressure sister balloon, is the ability to fly at an approximately constant elevation through the diurnal cycle. This stable platform, achieved without the need for dropping ballast, provides an ideal tool for scientific measurements. However, the introduction of pressure to the sealed system introduces design challenges and complications not present in the zero pressure balloons.

The SPB development project commenced in 1997 and suffered from a number of early failures related to construction methods and shape stability. Since 2004 there has been a rigorous evolution of the balloon design, supported by a program of finite element analysis, material characterization and scaled model tests.

Tensys have been involved throughout this period providing consulting support to NASA for stress and stability analysis, during which time the complexity of the finite element models and the functionality of the inTENS software, have advanced extensively to keep pace with project driven demands ${ }^{1-6}$. This paper introduces a number of recently undertaken analysis tasks, driven by a need to replicate and quantify recorded in flight phenomena and a requirement to better understand and model the structural system.

The analysis tools employed have been described previously. Their development has been greatly helped by the combined input of the SPB design and analysis team ${ }^{7-14}$.

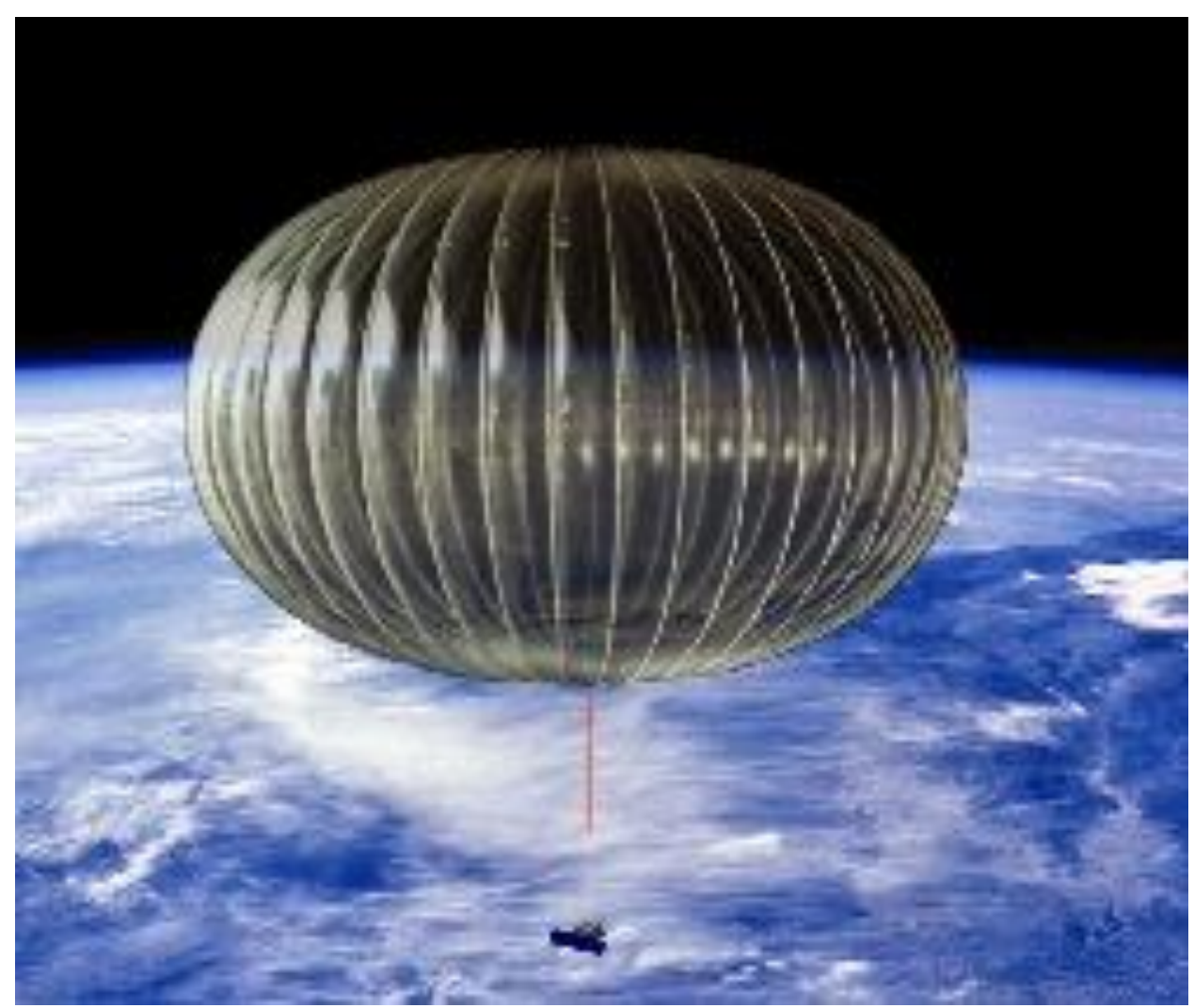

Figure 1. NASA SPB 


\section{B. Finite Element Model Review}

The finite element models comprise triangular elements for the balloon film and line tension elements to represent the tendons and seams/sleeves. The cap, an additional layer of film on the upper portion of the balloon to resist launchinduced stresses, is represented either by factoring of local film thickness for full balloon models or by a separate layer of contact controlled elements when undertaking more detailed local analyses. The end fittings are represented by stiff assemblies of strut elements which have been assigned appropriate self-weights to reflect the mass of the end fittings and attached flight control equipment. A point load representing the mass of the scientific payload is applied to the base fitting. A unit lift term is applied in addition to the base pressure value to account for the buoyancy effects of the enclosed helium.

Current balloons have in excess of 200 gores separated by tendons. Each gore is fabricated from a single flat piece of film, and this unstressed pattern geometry is mapped onto the numerical model to ensure a correct equilibrium shape upon inflation. See Figure 2 below.

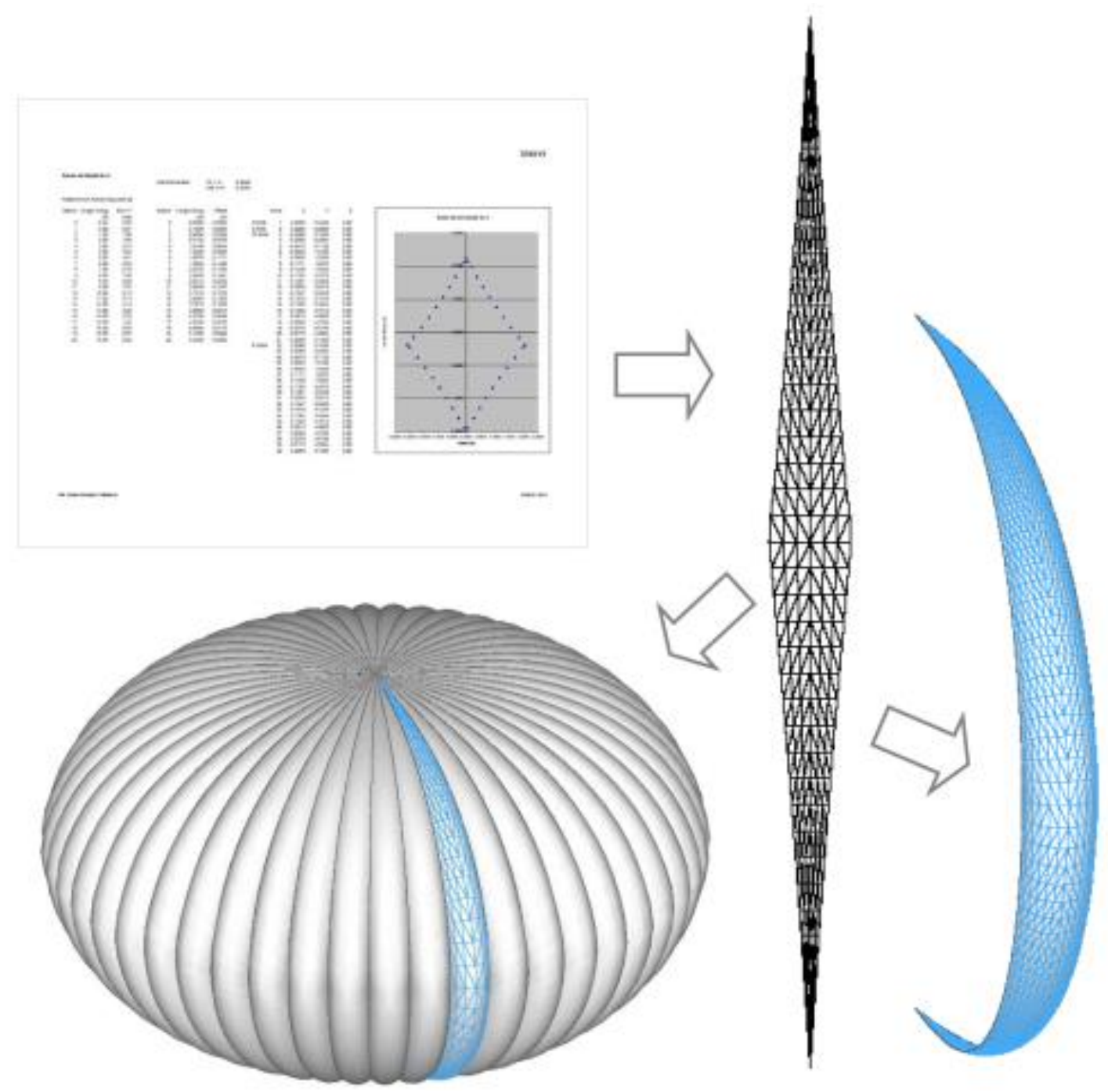

Figure 2. Mapping fabricated pattern geometry onto numerical model 


\section{Numerical Modelling}

\section{A. Dynamic Relaxation}

The inTENS finite element program suite for stressed membrane structure design has been under continual development by Tensys since 1990. It is based upon the Dynamic Relaxation (DR) solution technique that has particular advantages for this class of problem. Program modules are available for model generation, stress controlled equilibrium shape determination, load analysis, and membrane patterning ${ }^{2}$.

The static solution of both linear and non-linear structures subject to load may be regarded as the limiting equilibrium state of damped structural vibrations excited by that load. The physical basis of Dynamic Relaxation was initially perceived as the step-by-step solution, for small time increments $\Delta t$ of Newton's Second Law of Motion applied to a loaded structure subjected to an imposed viscous damping.

The basic iterative equations for the motion any node in space at time $t$ are obtained directly from Newton (Force=Mass x Acceleration) :

$$
\mathrm{R}^{\mathrm{t}}=M A^{\mathrm{t}}
$$

which may be expressed in central difference form

$$
\mathrm{R}^{\mathrm{t}}=\mathrm{M}\left(\mathrm{V}^{\mathrm{t}+\Delta \mathrm{t} / 2}-\mathrm{V}^{\mathrm{t}-\Delta \mathrm{t} / 2}\right) / \Delta \mathrm{t}
$$

giving the recurrence relation for nodal velocities :

$$
\mathrm{V}^{\mathrm{t}+\Delta \mathrm{t} / 2}=\mathrm{V}^{\mathrm{t}-\Delta \mathrm{t} / 2}+\Delta \mathrm{t} \mathrm{R}^{\mathrm{t}} / \mathrm{M}
$$

where $\quad \mathrm{R}^{\mathrm{t}}$ is the node residual force at time $\mathrm{t}$

$\mathrm{V}^{\mathrm{t}}$ is the associated node velocity

$\mathrm{M}$ is the node mass and $\Delta \mathrm{t}$ the time interval

The residual forces $\mathrm{R}^{\mathrm{t}}$ were computed for the then current node co-ordinates $\mathrm{X}^{\mathrm{t}}$. An updated set is calculated from the incremented node coordinates:

$$
\mathrm{X}^{\mathrm{t}+\Delta \mathrm{t}}=\mathrm{X}^{\mathrm{t}+\Delta \mathrm{t}}+\Delta \mathrm{t} \mathrm{V}^{\mathrm{t}+\Delta \mathrm{t} / 2}
$$

The original need to compute an optimized viscous damping coefficient has been eliminated by the adoption of a kinetic damping procedure. When an oscillating body passes through a local approximation to its static equilibrium position, then the plot of total kinetic energy against time passes through a local maximum. The total kinetic energy is traced as undamped iterations proceed, and all current node velocities are reset to zero whenever an energy peak is detected. The analysis continues, progressively eliminating the kinetic energy from various modes of vibration until the required degree of convergence is obtained. The process is automatic, requires no specification of damping factors, and can handle gross out of balance forces without the need for additional constraints. This means that gross geometrical changes and stiffness modifications may be accommodated during an analysis.

On first inspection this might seem a rather long-winded approach, but it does have significant advantages when applied to the design of tension structures:

1) An explicit solution technique - a time-stepping dynamic analysis with automated kinetic energy damping control. The static solution to an applied loading case is obtained by damping out a dynamic application of that load.

2) A natural treatment of large deformations coupled with a tolerance of the very large out-of-balance forces that are often associated with significant geometric non-linearity.

3) Computer storage requirements increase linearly with problem size, and the computations are all held within memory rather than requiring significant interaction with storage devices. Surprisingly large problems may be tackled on conventional PC hardware. 
4) Needing only individual element natural stiffnesses without the formation of an overall structure matrix, it is straightforward to introduce on-off non-linearities such as cable slackening and membrane wrinkling. Similarly a wide range of nodal constraints is possible. Specified element stress states may also be introduced, such as for the shape generation of membrane surfaces where the final geometry may be adjusted by continually varying stresses in the fabric weave directions.

5) A full range of elements is available to facilitate the modeling of complete structures, including membranes, cables, struts and beams.

6) By using large numbers of simple elements within a method founded on a clear physical analogy, the interpretation of results and unforeseen occurrences is facilitated.

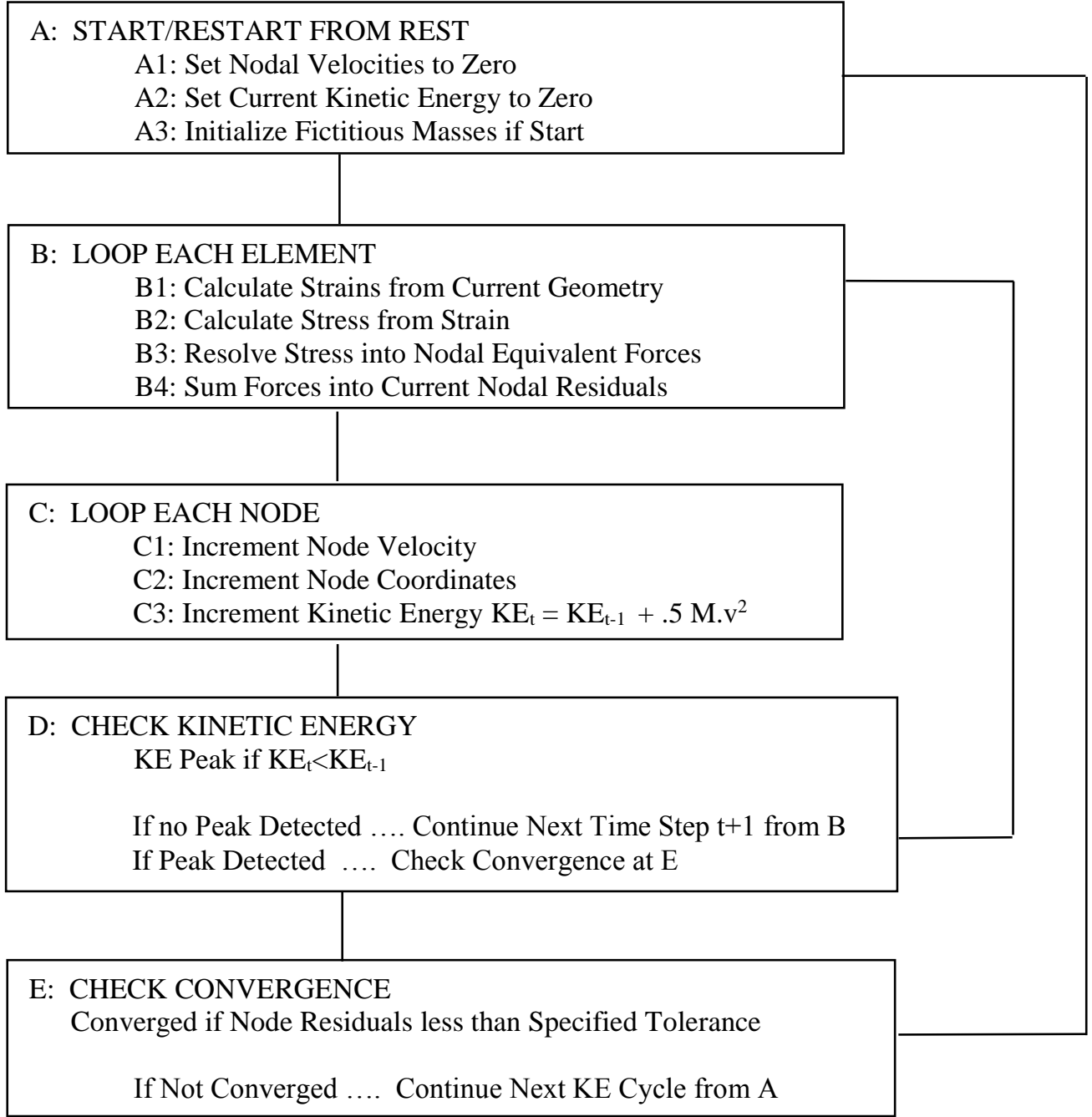

Figure 3. Dynamic Relaxation Flowchart 
Note that

1) An overall stiffness matrix is not required.

2) Checks on membrane wrinkling are applied individually at stage B2.

3) Non-linear stress strain relations are introduced by revised code for stage B2.

4) Geometric non-linearity and rigid body movement handled automatically as all strain calculations and element force resolution based upon current updated coordinates.

5) Time steps are fictitious and node masses determined to optimize convergence. Kinetic energy damping results in a static solution to particular applied load state.

Although initially developed for architectural fabric structure applications ${ }^{5}$, the program has been progressively extended for broader applications. Other applications include large kites, such the SkySails system for wind power assisted propulsion of cargo ships and hybrid airships ${ }^{6}$.

\section{B. Pumpkin Balloon Modelling}

The relative storage and computational efficiency of DR as used in inTENS makes it practical to analyze complete balloon models built from several hundred thousand nodes and elements on standard PC hardware. The models themselves comprise constant strain triangular elements for the balloon film, line tension elements to represent the tendons and an assemblage of struts to model the stiff capping plates at base and apex.

Given the size and symmetry of the structures an automated data generator was written to efficiently produce the standard inTENS data files. These generated an initially spherical balloon shape to which inflation pressure was applied, together with specified stresses in the membrane, to establish a preliminary equilibrated pumpkin shape with the correct tendon lengths and an approximate profile of the lobed film between those tendons ${ }^{5}$.

In practice each gore of the balloon between the tendons is fabricated from a single flat piece of polyethylene film. In order to correctly model the as-built structure, this flat pattern geometry is then mapped onto the preliminary mode (Fig. 4) which must then be reanalyzed to establish a correct equilibrium stressed shape.

To fully represent a flight balloon, additional features must be added to the model. The cap, an additional thinner layer of film on the upper portion of the balloon to resist launch-induced stresses, may be approximately represented by factoring of local stiffnesses.

The end fittings are represented by stiff assemblies of strut elements and appropriate self-weights are applied, along with the payload and the buoyancy effects of the enclosed gas. The load tape tendons are modeled as linear elastic tension elements with an initial strain, according to load/strain data generated by the fabricator, Raven Aerostar, as part of the development of tendon pre-processing procedures.

The most significant analytic difference between a flight balloon and its counterpart ground model is the influence of temperature. Significant additional stresses in the meridional direction are generated because of the differential between the coefficients of thermal expansion (CTE) between the film and the PBO tendons. The latter exhibit effectively no thermal strain, in comparison to the film that has differing values in machine and transverse direction, and values that themselves vary with temperature

\section{Fine Mesh Modeling of Balloon Details}

A film rupture near the apex fitting of a balloon envelope as it reached float elevation over Antarctica in early 2010 highlighted the need to consider the low pressure, low temperature conditions associated with initial deployment and also improve the detail of the FE models in the vicinity of the end fittings. The analysis of subsequent balloons has included a fine mesh representation of the zones adjacent to the end fittings, with full representation of the effective stiffness of the various seam, tendon sleeve and local taped collar reinforcement components in those areas. Their additional stiffnesses are included in the model by local thickening of the line elements representing the seam assembly (Fig. 4). 


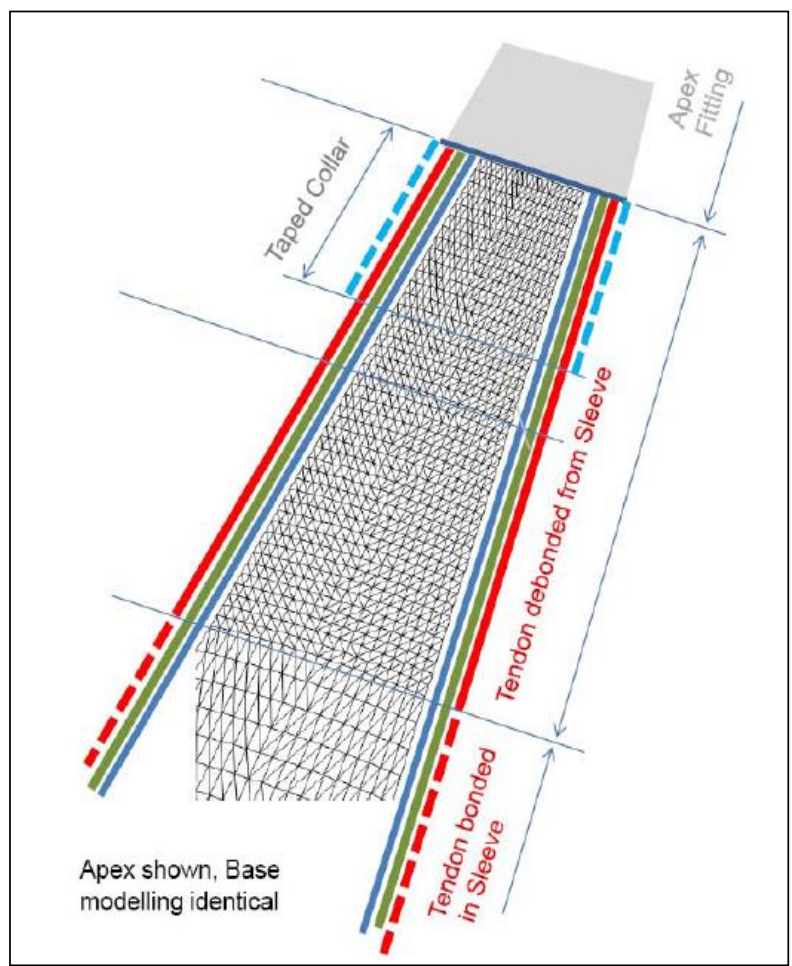

Figure 4a. Schematic of additional detail included in a single gore fine mesh model to take into account the various film layers adjacent to the end fittings.

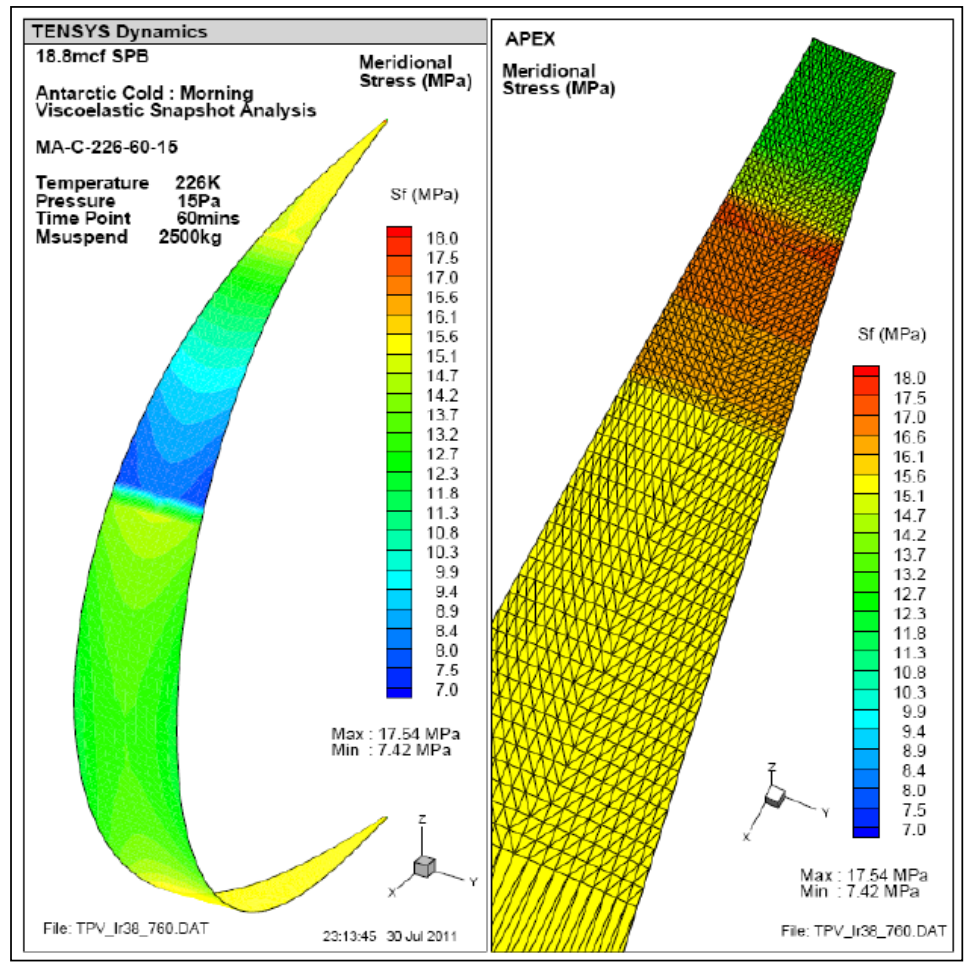

Figure 4b. Stress plots from those areas for the nominal design case of an $18.8 \mathrm{mcf}$ balloon

American Institute of Aeronautics and Astronautics 


\section{Contact and Analysis of Cap}

To gain a more accurate picture of the stress fields and tendon/sleeve interaction at the location where the cap terminates, models with a reduced number of gores were developed with the second, cap, layer of film modeled independently from the underlying envelope. This second layer of film was controlled to interact with the main envelope via a simplified contact procedure (Fig. 5).

General contact algorithms had previously been considered for inclusion into inTENS but such tools rapidly become computationally expensive since each node (of potentially several hundred thousand in these particular models) has to be checked for potential contact interactions with all other nodes. Since the cap is connected along the common edge of the main gores, the degree of relative movement between the cap and underlying gore is limited. The location of the nodes forming the cap are restricted to a small area and can therefore be associated with a single underlying node from the main gore. This limited contact specification facilitated contact functionality in inTENS which allows the cap to freely slide over surface the main gore with only minimal effect on run times.

The inclusion of the cap as a separate entity permits a more accurate representation of film stresses at cap drop area. The previous method of thickening film would, under certain loaded conditions, result in a small hot spot of peak stresses at cap drop area where the film thickness reverts to a single layer. Figure 6 shows such a typical stress 'hot spot' on an old single gore model compared with the more accurate stress field on a five gore model where the cap has been modelled separately.

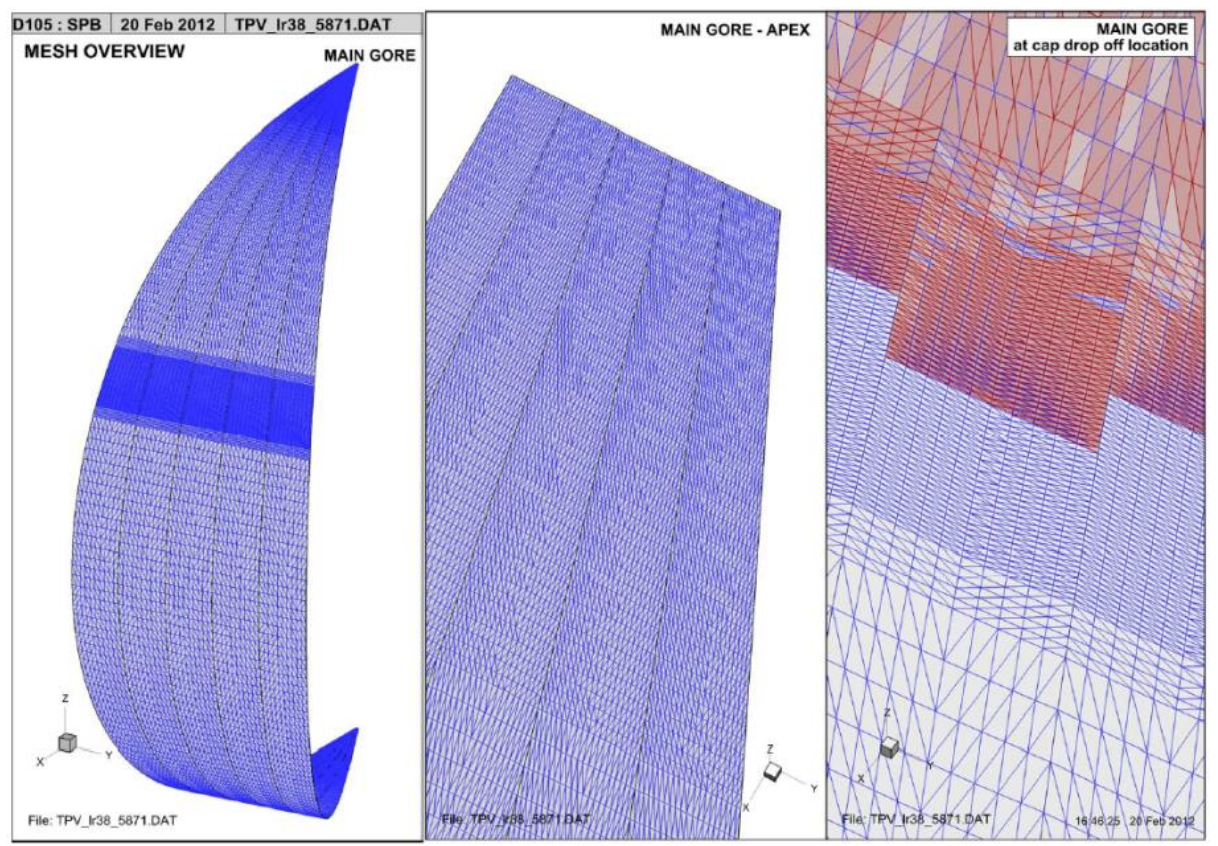

Figure 5. Increase in fidelity of FE mesh at the location of the cap drop (LHS) and cap modeled as a separate layer of film resting on top of the underlying gore film (orange layer on RHS) 

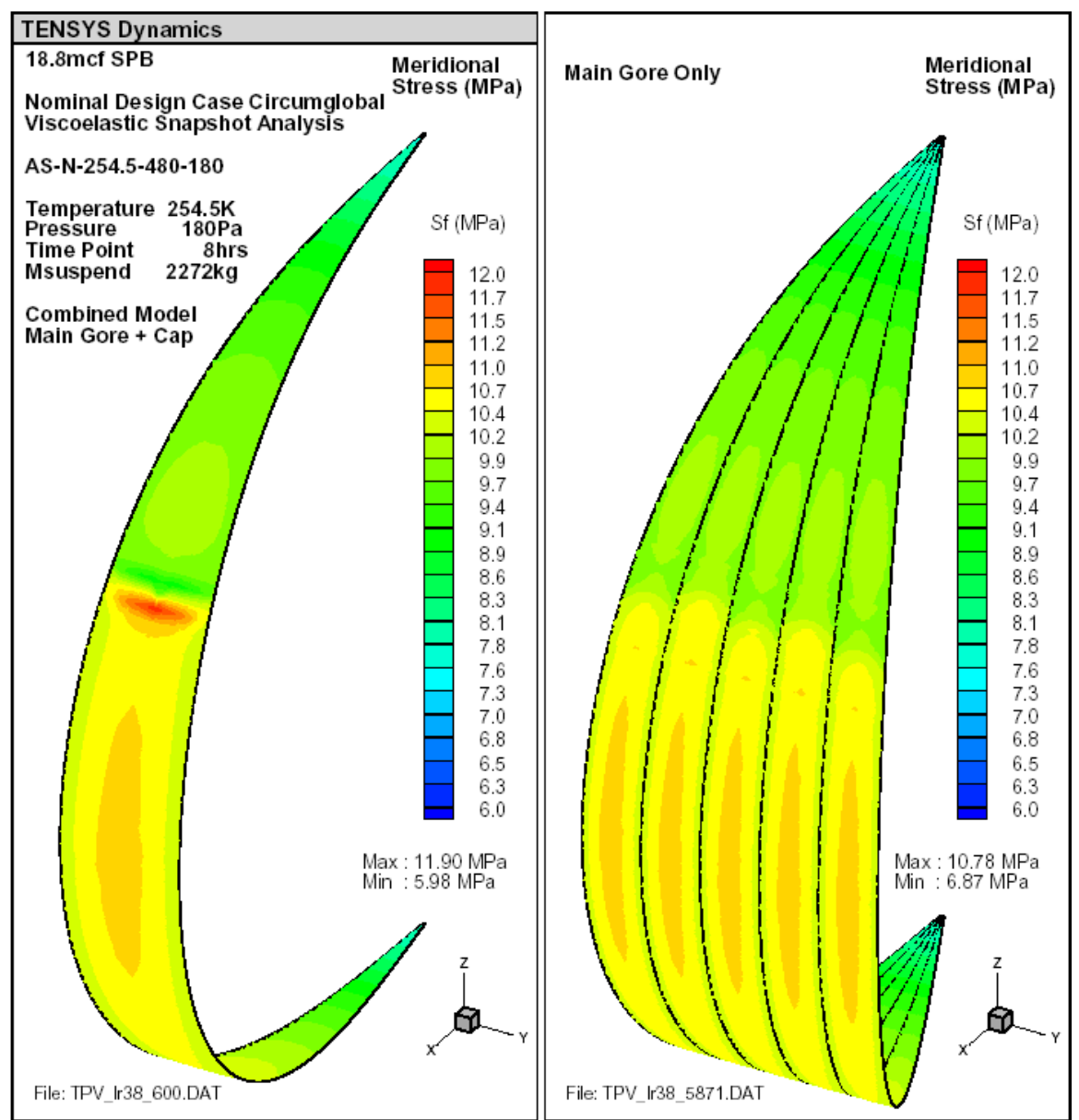

Figure 6. Meridional stresses in fine mesh model with cap represented by film thickening (LHS), and as separate layer (RHS). Note the disappearance of the peak stress at the cap drop location.

Figure 7 shows typical meridional and hoop direction stresses in the cap from the five gore model. Note the reduction in meridional direction stresses towards the end of the cap. The cap edge is not connected to the underlying film and therefore bows in slightly to form a catenary when the lobe is loaded.
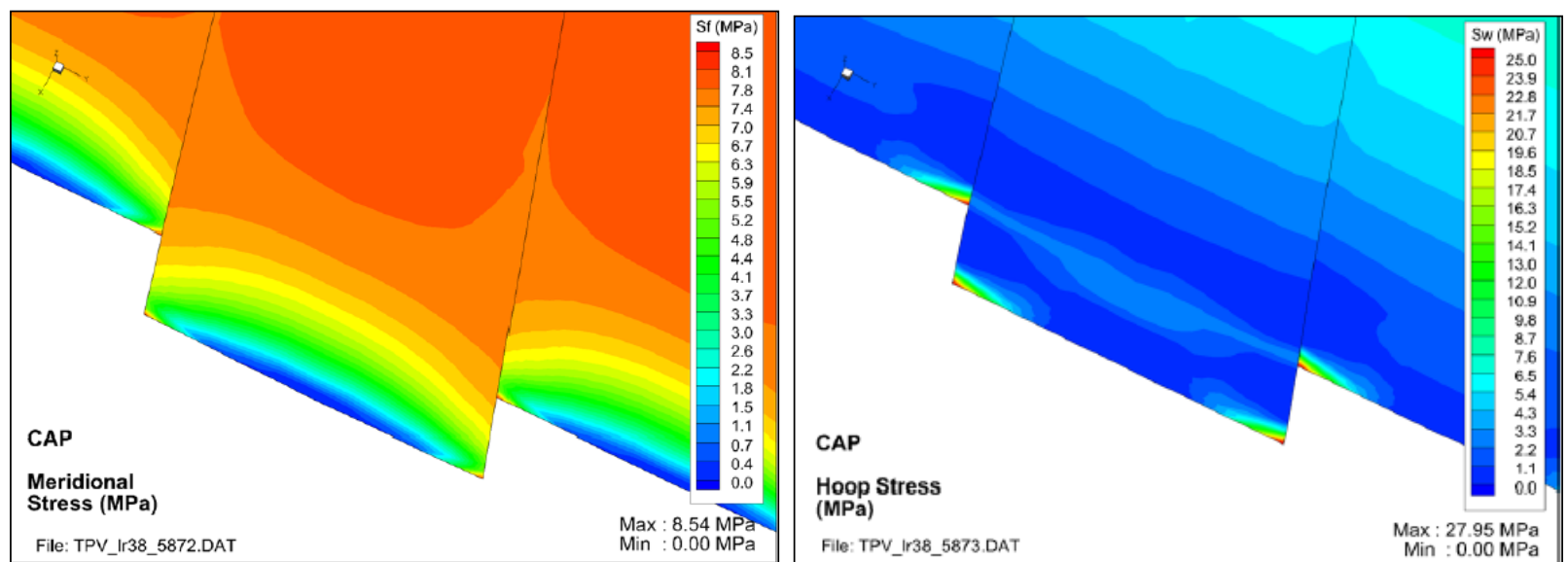

Figure 7. Typical stresses in the cap layer only.

American Institute of Aeronautics and Astronautics 


\section{Material Idealisation}

\section{A. Introduction to Current Design Model}

The design of the NASA super pressure balloons is based upon the Schapery-Rand material model described in earlier papers ${ }^{7,8}$. This has been implemented in the balloon design, patterning and performance modelling tools by Farley at NASA Goddard Space Flight Centre ${ }^{13}$. It is also implemented in the Tensys inTENS software suite that has been used for detailed finite element analysis in support of the design tools.

The results of the analyses are compared against yield stress design criteria based upon film creep and yield testing.

\section{B. Schapery Rand Model: Nonlinear Material Model for Snapshot Analysis}

A creep compliance model is used to characterize the non-linear behaviour of the film ${ }^{8}$. The linear viscoelastic creep compliance $D_{\psi}$ is defined as the strain response for a unit stress. The current compliance at any time comprises an elastic component $D_{0}$, which is independent of stress and temperature, and a transient component $\Delta D(\psi)$ where $\psi$ is the reduced time that incorporates the effects of stress and temperature:

$$
D_{\psi}=D_{0}+\Delta D(\psi)
$$

The transient component may be expressed as a series of exponentials in reduced time:

$$
\Delta D(\psi)=\sum_{r=1}^{N} A_{r}\left(1-e^{-\lambda_{r} \psi}\right) \quad \text { where } \lambda_{r}=10^{a-b . r}
$$

For biaxial compliance it is has been shown that the transient compliance in any direction may be expressed in terms of a constant multiplied by the compliance in the machine direction:

$$
\Delta D_{i j}=S_{i j} \Delta D
$$

The three strains due to constant biaxial stress may be expressed:

$$
\begin{aligned}
& \varepsilon_{1}=D_{0}\left(\sigma_{1}+S_{12}^{0} \sigma_{2}\right)+g_{2} \cdot \Delta D(\psi)\left(\sigma_{1}+S_{12} \sigma_{2}\right) \\
& \varepsilon_{2}=D_{0}\left(S_{21}^{0} \sigma_{1}+S_{22}^{0} \sigma_{2}\right)+g_{2 .} \Delta D(\psi)\left(S_{21} \sigma_{1}+S_{22} \sigma_{2}\right) \\
& \varepsilon_{6}=D_{0} S_{66}^{0} \sigma_{6}+g_{2} \cdot \Delta D(\psi) S_{66} \sigma_{6}
\end{aligned}
$$

where $g_{2}$ is a function of stress for the non-linear viscoelastic realm

This creep compliance model predicts film strains for a stress state that has been applied for a given length of time and at a particular temperature. Thus an equivalent elastic modulus can be derived that would predict the same strains when used with the same stress state. It should be emphasized that this is not the current, or tangent, stiffness.

\section{Schapery Rand Model: Snapshot Analysis Sequence}

A snapshot analysis is one that captures the state of stress and strain at a defined elapsed time. The other inputs necessary for the full definition of the loading conditions are the temperature and pressure distributions at that time. It is characterized as an iterative solution of the equilibrium of the balloon at that elapsed time. The iterations involve updating of the viscoelastic material properties until the current stresses calculated for the strained state of the balloon are equal to those used for the derivation of those properties.

Viscoelastic material properties are assigned individually to all membrane elements, representing the main gore film, and line elements representing the localised additional stiffness of seams and load tendon sleeves. These properties are initially assigned according using element stresses that are either taken from the results of a previous analysis or supplied as input data. These initial estimates need not be highly accurate as they are progressively updated 
by the iterative analysis sequence. The PBO tendons are modeled as linear elastic with initial strains, with the values used being established from tests undertaken by the balloon fabricator Aerostar.

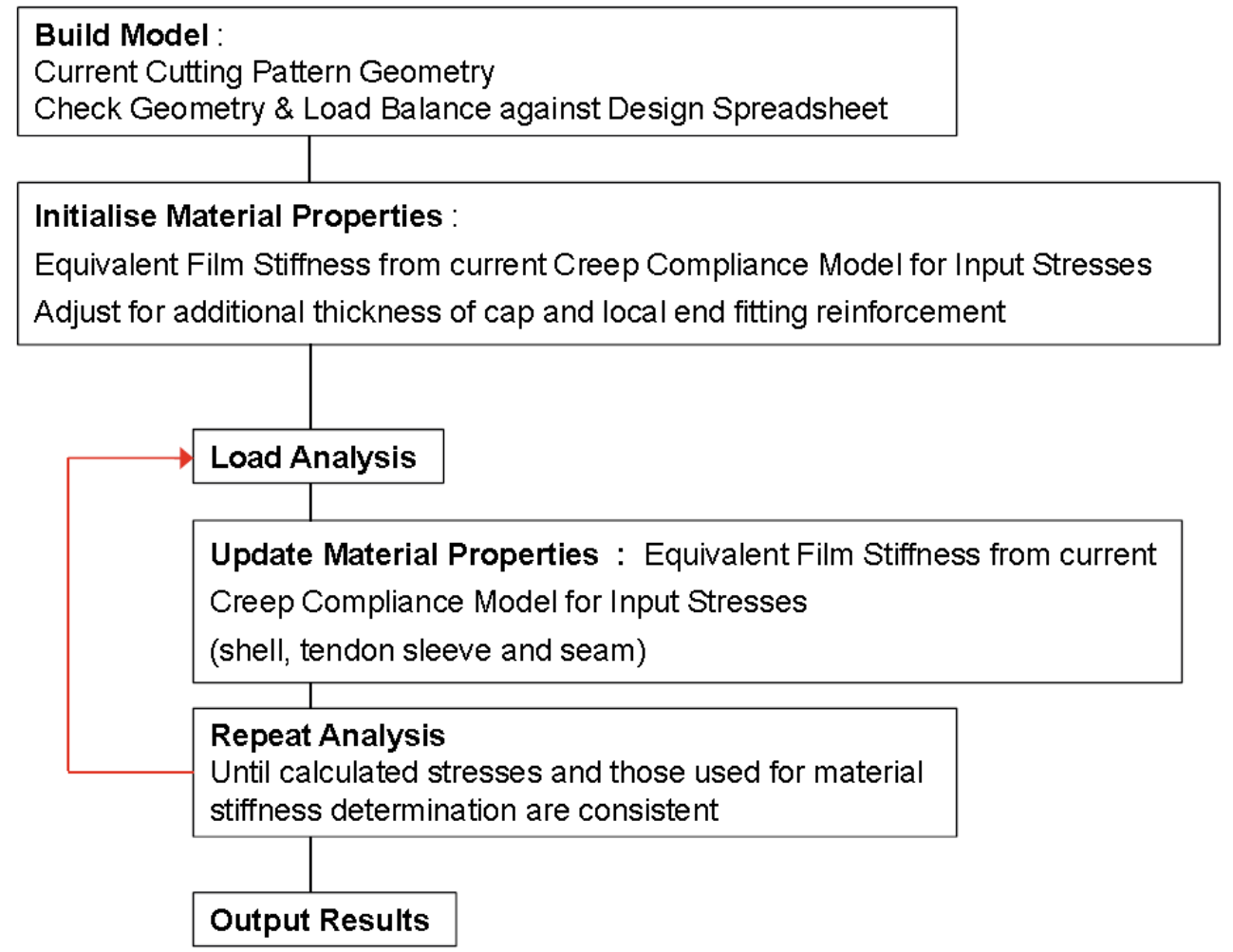

Figure 8. Snapshot Analysis Sequence

Typically 4 or 5 analysis cycles prove sufficient to obtain a solution with balanced stresses and material properties.

\section{Large Strain Material Model}

\section{A. Development of the large strain non-linear viscoelastic model}

This model was initially developed at Caltech by Kwok and Li under the supervision of Professor Sergio Pellegrino ${ }^{15}$ with support of the NASA Balloon Program Office. This work has recently been extended by Bosi ${ }^{17}$ to cover the wider range of operational conditions the material will experience during flight. A longer time domain with slower deformation rates and a wider temperature range has been considered together differing loading conditions including relaxation and cyclic tests. Professor Pellegrino and his co-workers are long term members of the Super Pressure Balloon development team.

The model has been developed as a natural extension of the Rand model discussed above. The prime aims were to establish a model capable of accurately replicating the large strain behavior of the balloon material and also enabling full time-stepping analyses capable of handling the range of temperature and stress variations during flight. The large strain capability is seen as particularly important when considering the performance of localized details and investigating the likely dissipation of stress concentrations. A refined assessment of the structural resilience of the balloon system will include the beneficial aspects of viscoelastic response in facilitating this dissipation. A viable time-stepping analysis capability is the other necessary component of such analyses. Whilst the Rand model remains a key tool for the overall assessment of the basic balloon design, the new model allows the team to better consider the fine details of the construction. With the program moving towards long duration flights the need to efficiently assess long-term behavior also becomes more important. 
The first stage of development of the model focused on linear viscoelasticity. When assessing the results of DMA and creep tests the SF420 film was shown not to be thermo-rheologically 'simple', that is when considering timetemperature superposition the time shift factors were not the same for compliance, modulus and damping variables. Creep tests were chosen as the basis for the ongoing derivation of the model as they were deemed closer to the actual structural problem in hand. The model was developed for a compliance formulation, with subsequent matrix inversion being utilized for the modulus or relaxation formulation.

Building upon this, the large strain non-linear viscoelastic capability was founded on a free volume model which accounted for non-linear effects by considering the out-of-plane response of the film. Initial versions worked well for uniaxial behavior but less so for biaxial response. An extension of the free volume model was utilized that included an allowance for distortion through the inclusion of an effective shear strain term in the expression for the time shift factor. The model showed good correlation with both bubble and cylinder tests undertaken at Caltech and the NASA Balloon Research and Development Laboratory at Wallops Island VA.

The subsequent development of the model and the associated test procedure is comprehensively described by Bosi and Pellegrino ${ }^{17}$. The model was implemented within Abaqus/Standard by Bosi through a user-defined material subroutine (UMAT). The summary here is intended to place that development in the context of a particular project requirement and the interaction between the materials, design and analysis groups within the team.

From the Tensys perspective the prime goal was to enable a viable time-stepping solution within our inTENS finite element code. This would support both flight duration overall analyses and detailed studies of stress concentrations and their relaxation at particular construction details. It was also hoped to implement an enhanced snapshot procedure for the efficient investigation of specific loading cases. The latter was first described in our last paper ${ }^{19}$ but with limited success at that time

\section{B. Incremental Analysis}

A basic flow chart for the time stepping or incremental analysis is shown in figure 9.

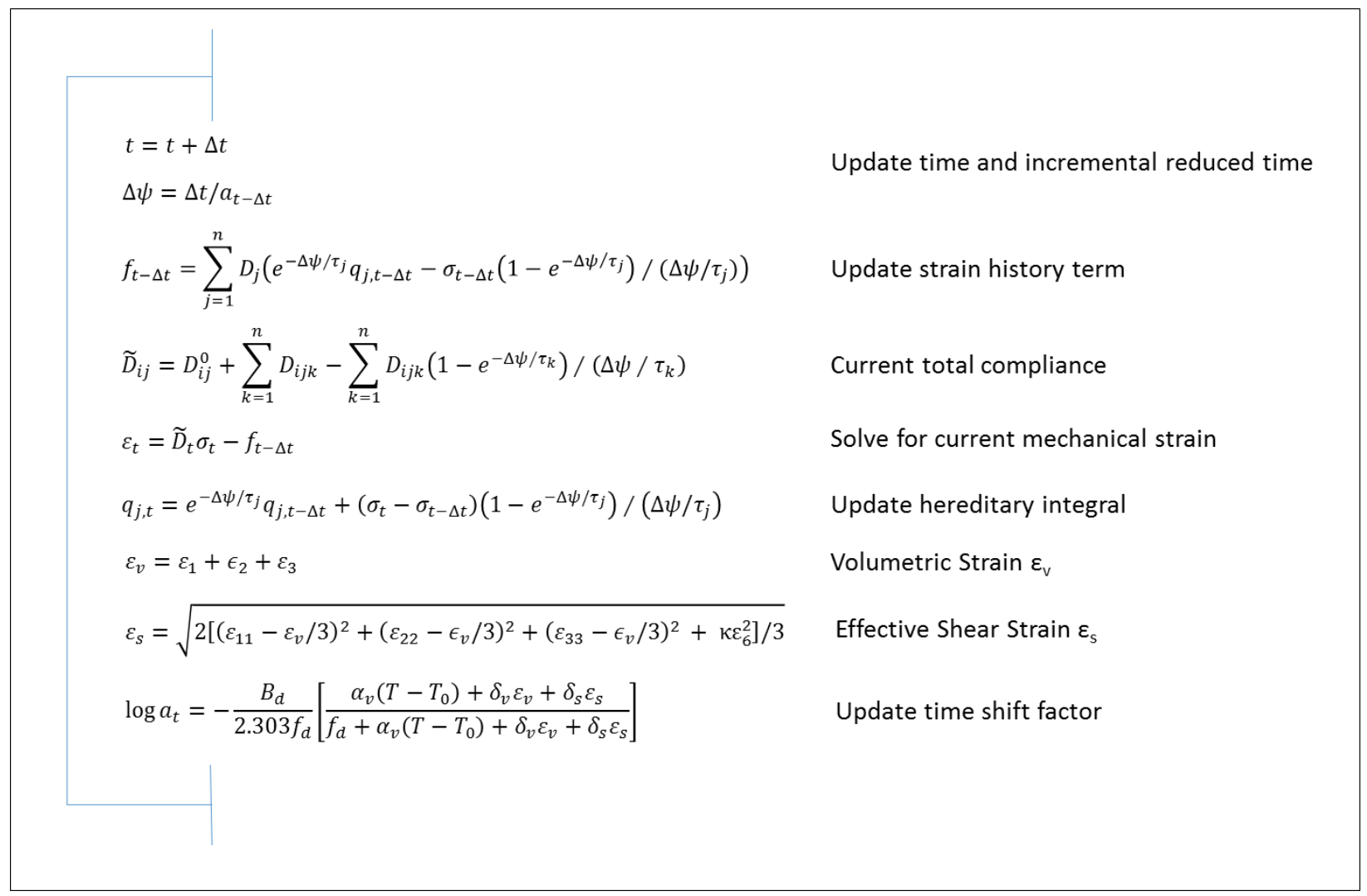

Figure 9. Large Strain Model - Flow Chart for Incremental Analysis 


$$
\begin{aligned}
& {\left[\begin{array}{l}
\grave{\varepsilon}_{1} \\
\grave{\varepsilon}_{2} \\
\grave{\varepsilon}_{3} \\
\grave{\varepsilon}_{6}
\end{array}\right]=\left[\begin{array}{cccc}
D_{11}^{0} & D_{12}^{0} & D_{13}^{0} & 0 \\
D_{21}^{0} & D_{22}^{0} & D_{23}^{0} & 0 \\
D_{31}^{0} & D_{32}^{0} & 0 & 0 \\
0 & 0 & 0 & D_{66}^{0}
\end{array}\right]\left[\begin{array}{c}
\sigma_{1} \\
\sigma_{2} \\
0 \\
\grave{\sigma}_{6}
\end{array}\right]} \\
& {[\grave{\varepsilon}]=\left[D_{o}\right][\grave{\sigma}] \quad \text { Elastic Compliance }} \\
& {\left[\begin{array}{l}
\varepsilon_{1} \\
\varepsilon_{2} \\
\varepsilon_{3} \\
\varepsilon_{6}
\end{array}\right]_{t}=\left[\begin{array}{cccc}
\widetilde{D}_{11} & \widetilde{D}_{12} & \widetilde{D}_{13} & 0 \\
\widetilde{D}_{21} & \widetilde{D}_{22} & \widetilde{D}_{23} & 0 \\
\widetilde{D}_{31} & \widetilde{D}_{32} & 0 & 0 \\
0 & 0 & 0 & \widetilde{D}_{66}
\end{array}\right]\left[\begin{array}{c}
\sigma_{1} \\
\sigma_{2} \\
0 \\
\sigma_{6}
\end{array}\right]_{t}-\left[\begin{array}{c}
f_{11}+f_{21} \\
f_{22}+f_{12} \\
f_{13}+f_{23} \\
f_{66}
\end{array}\right]_{t-\Delta t}} \\
& \varepsilon_{t}=\widetilde{D}_{t} \sigma_{t}-f_{t-\Delta t} \quad \text { Total Compliance at Time t } \\
& \widetilde{D}_{i j}=D_{i j}^{0}+\sum_{k=1}^{n} D_{i j k}-\sum_{k=1}^{n} D_{i j k}\left(1-e^{-\Delta \psi / \tau_{k}}\right) /\left(\Delta \psi / \tau_{k}\right) \\
& \Delta \psi \quad \text { Increment of reduced time } \\
& \tau_{k} \quad \text { Prony Series retardation time } \\
& n \quad \text { Order of Prony Series } \\
& D_{i j k} \quad \text { Prony Series terms for ij coefficient } \\
& {\left[\begin{array}{l}
\sigma_{1} \\
\sigma_{2}
\end{array}\right]_{t}=\left[\begin{array}{ll}
\widetilde{D}_{11} & \widetilde{D}_{12} \\
\widetilde{D}_{21} & \widetilde{D}_{22}
\end{array}\right]_{t}^{-1}\left[\begin{array}{l}
\varepsilon_{1}^{t}+f_{11}^{t-\Delta t}+f_{21}^{t-\Delta t} \\
\epsilon_{2}^{t}+f_{12}^{t-\Delta t}+f_{22}^{t-\Delta t}
\end{array}\right]} \\
& \text { Inverse case when strains } \epsilon_{1}, \epsilon_{2} \text { and } \epsilon_{6} \text { are known } \\
& \sigma_{6}^{t}=\left(\varepsilon_{6}^{t}+f_{6}^{t-\Delta t}\right) / \widetilde{D}_{66} \\
& \varepsilon_{3}^{t}=\widetilde{D}_{31} \sigma_{1}^{t}+\widetilde{D}_{32} \sigma_{2}^{t}-f_{13}^{t-\Delta t}-f_{23}^{t-\Delta t}
\end{aligned}
$$

Figure 10. Large Strain Model - Compliance terms in Matrix Notation

\section{Snapshot Analysis and the Caltech Large Strain Model}

Tensys have previously undertaken 'snapshot' analyses for a particular temperature, pressure and elapsed time using the Schapery Rand material model. These calculations assume the final stress state has been in place for the duration of the elapsed time, with equivalent elastic properties determined for these parameters. These are single analyses, not an incremental sequence, but they do feature iterative updating of these equivalent properties because of their stress dependent non-linearity. This approach has the advantage of requiring significantly less computational time, especially when features such as modelling separate cap layers with contact are included
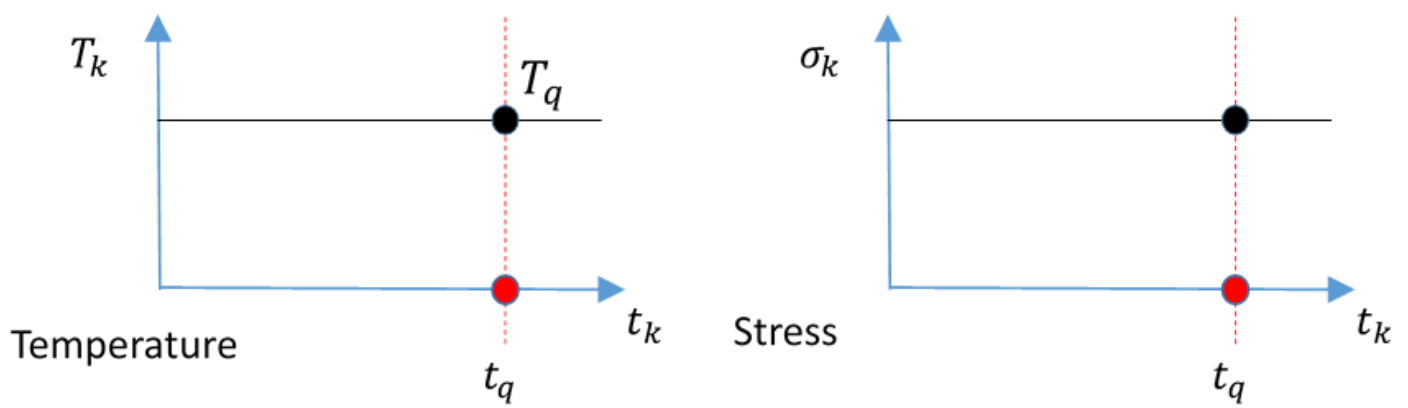

Figure 11. Stress and Temperature Assumptions for Schapery Rand Snapshot Analysis

Given a temperature, stress state and elapsed time it is not possible to predict the associated strains in a single iteration when using the Caltech large strain model. This is because the final solution is now dependent upon the strain 
history. This simply means that an incremental calculation must be used. The number of time steps and appropriate stress/time relationship will be established by numerical experiment.

The previous model produced a total compliance solution that could be directly inverted to obtain the equivalent elastic properties. The presence of the strain history term complicates matters for the new large strain model. With this in mind a new procedure has been adopted:

- An individual incremental computation of updated material properties is undertaken for each element having current stress $\sigma_{q}$ and temperature $T_{q}$ at elapsed snapshot time $t_{q}$

- At intermediate time points $t_{k}\left(0 \leq t_{k} \leq t_{q}\right)$ the temperature $T_{k}$ and stress $\sigma_{k}$ vary according to a predefined distribution. The stress is defined by $\sigma_{k}=S_{k} . \sigma_{q}$, where the stress factor $S_{k}$ has a value of 1.0 at the elapsed snapshot time $t_{q}$

- For a particular flight profile the stress factor profile could be based upon the parametric increase in pressure over time from launch to the target snapshot time. Typically a single factor is currently used for both MD and TD direction stresses, but separate factors have also been implemented.
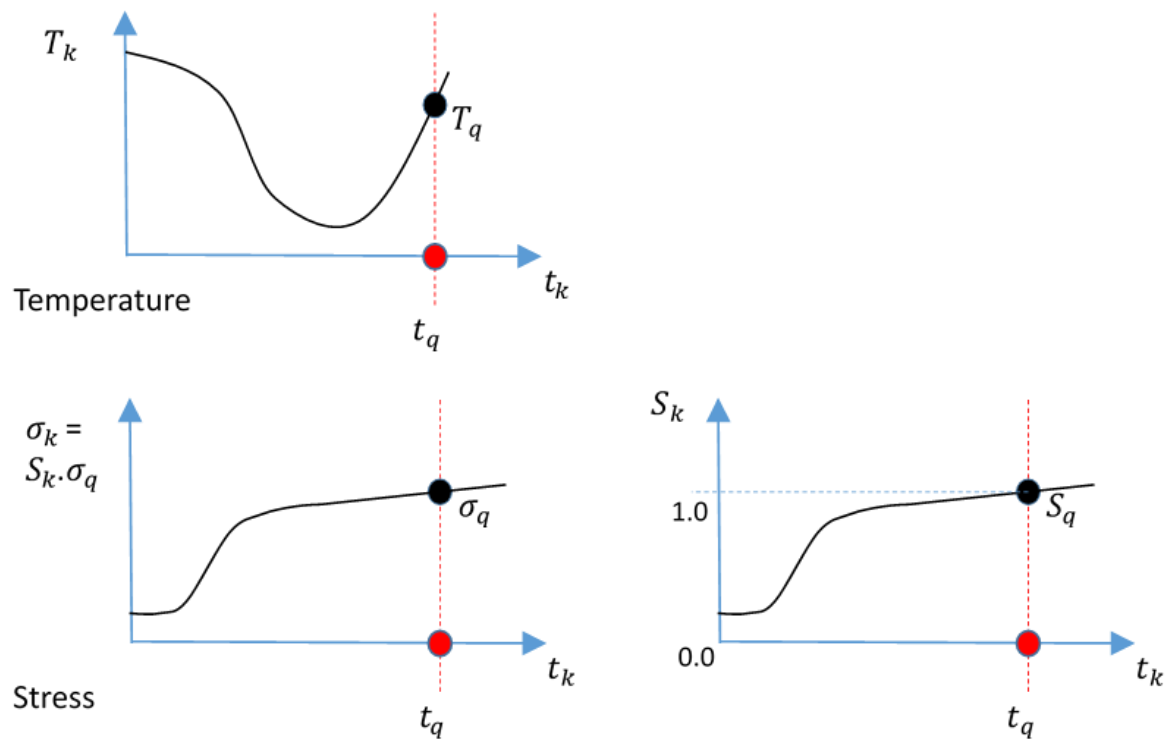

- The equilibrium analysis of the balloon is only solved at the snapshot time $t_{q}$

- The individual element material properties are updated after each solution according to predefined temperature and stress profiles

- The equilibrium analysis at $t_{q}$ is repeated until the property update has effectively no change

Previous studies ${ }^{19}$ showed good correlation between snapshot and incremental solutions for simple uni-axial and biaxial test examples but encountered convergence issues when applied to a section of a lobed balloon gore. The updated Caltech model has not made any difference to this outcome.

Subsequent effort has been focused on the full incremental time-stepping implementation within inTENS. This makes full use of the visco-elastic model without compromise. 


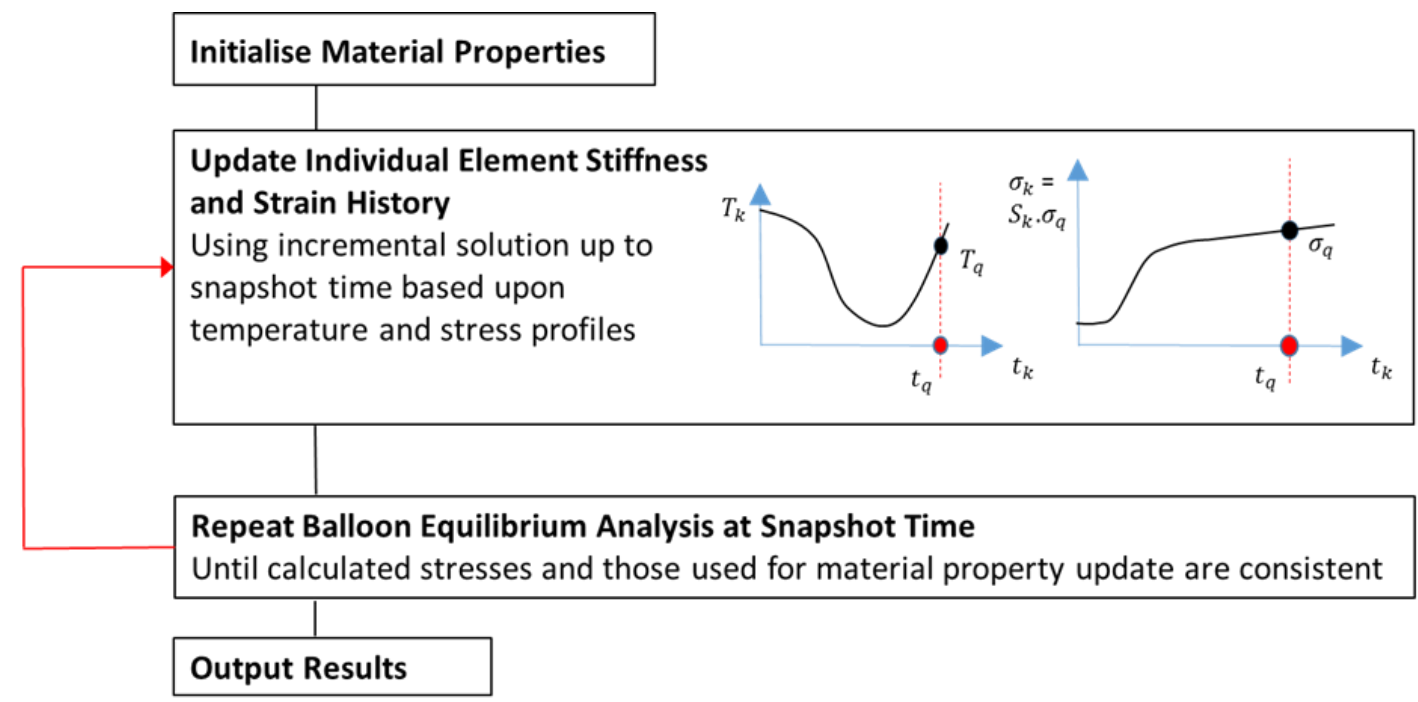

Principal Assumption:

A stress factor profile is defined for the time sequence up until the snapshot time. The individual element stresses during this time are assumed to be equal to the final snapshot stress multiplied by this factor.

Figure 12. Large Strain Model - Outline of Updated Snapshot Analysis Sequence

\section{Large Strain Model - Time-Stepping Incremental Analysis}

In the incremental implementation of the Caltech large strain model it has been shown that the analysis at any time point depends solely upon the current state and strain history and hereditary integral terms determined at the previous time step.

This is not only efficient in terms of computational storage requirements it also means that analyses can be restarted from any time point provided that the strain history and hereditary integral terms associated with that time have been retained. The latter forms the basis of the whole-flight simulations now undertaken for the SPB project.

\section{Whole Flight Simulations}

\section{A. Introduction}

The large strain visco-elastic model described above adds significant capability to the analysis of the super pressure balloon.

Previous design analysis has been based upon a snap shot implementation to estimate response at a particular assuming that the resultant stress state has been in place for the full duration of that time. This was used in the absence of a reliable time stepping implementation of the Schapery Rand material model. 


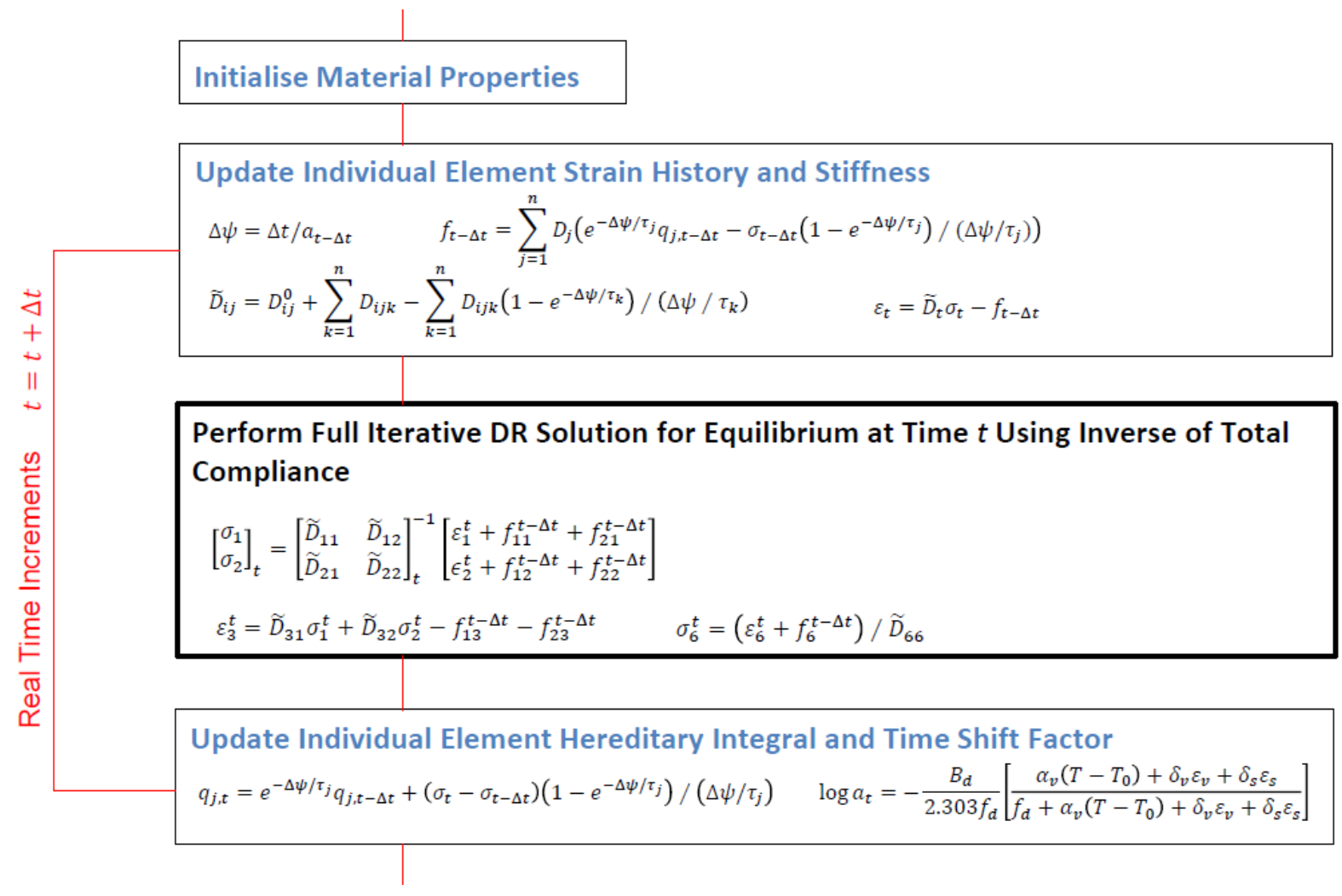

Figure 13. Large Strain Model - Time-stepping Incremental Analysis Flowchart

A similar snapshot approach was considered for the Caltech large strain model. This potentially had improved computational efficiency compared to the full incremental implementation and was an improvement on the previous model in that a variable temperature and stress history was assumed over the flight preceding the chosen analysis point. Convergence issues previously reported have not fully resolved. It had always been the intention to verify the updated snapshot approach by means of a full incremental solution. That full solution has now become the basis of flight analysis for the super pressure balloon.

The key to the approach adopted lies in the fact that all the prior time dependent effects can be encapsulated in a set variables updated at the end of a particular time step. The quantity of data required is the same for any time point independent of whether it lies early or late in a flight sequence. This data need not be stored for each time increment. For example, analyses are typically undertaken at 10 minute time increments whilst the history data is only stored every 12 or 24 hours.

The chosen procedure is to run a time stepping simulation using a repeating typical diurnal temperature and pressure sequence for the full flight duration, say 100 days with regular history data output as above. Specific nonstandard load cases, such as extremes of temperature and pressure, can then be run at any elapsed time in the flight by running that new load case using the associated history data as a start point. If necessary that new analysis can be followed by repeats of the typical loading sequence to assess the long term effects, if any, of that particular case.

With a full flight simulation requiring up to 15,000 individual equilibrium solutions, the computational requirement is not insignificant. Once the library of reference typical computations has been established however, then the individual cases can be run reasonably efficiently. This has proved an effective way of exploiting the full flight analysis capability. 


\section{B. Definition of Typical/Reference Flight Profile}

The basic set of SPB snapshot design load cases has been established by Farley using BalloonAscent ${ }^{13}$, the flight physics simulation tool.
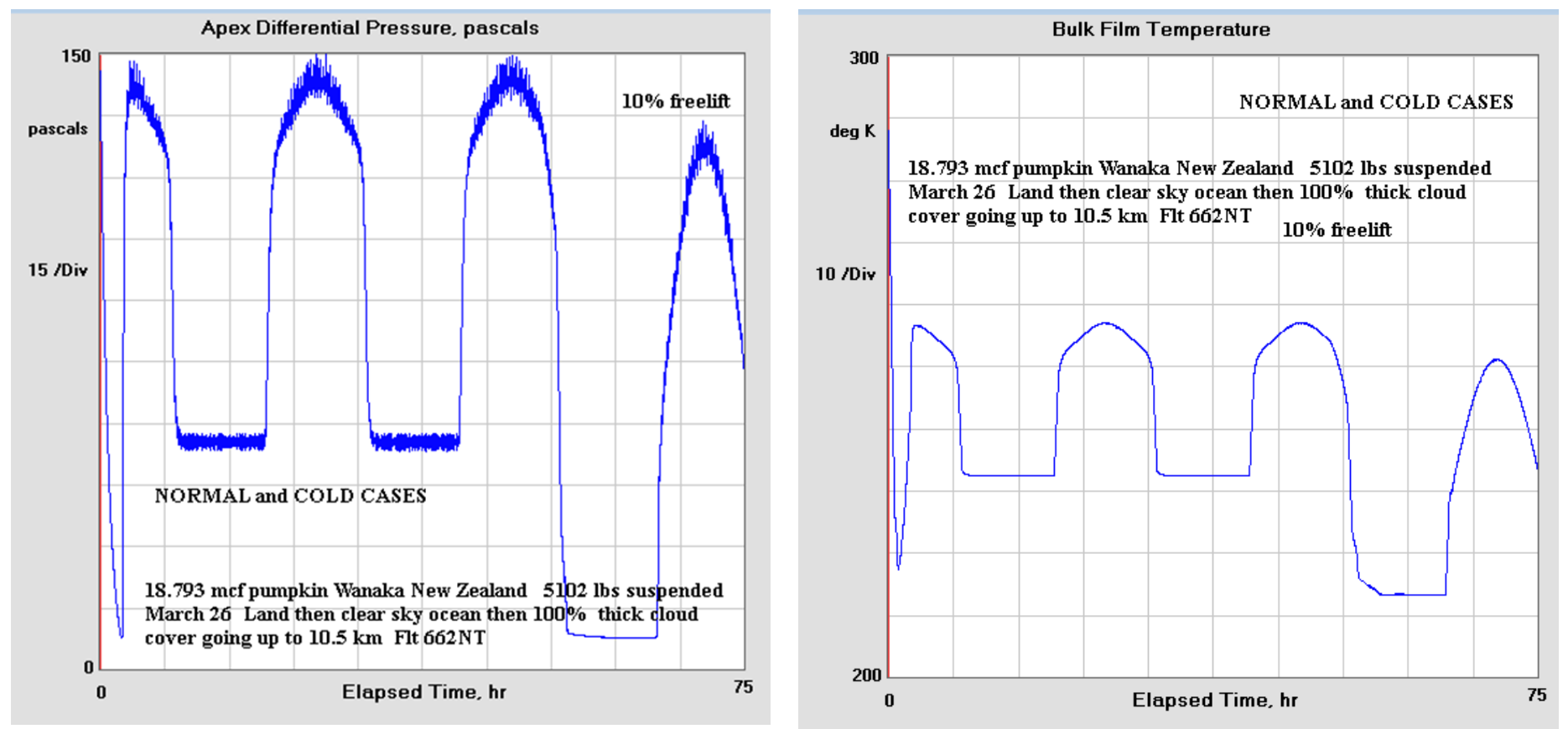

Figure 15. BalloonAscent Flight Physics Simulation - Typical Pressure and Temperature Output

BalloonAscent undertakes a transient analysis of balloon flight, predicting the skin, load tape, and gas temperatures along with the 3-D position and velocity in a time and spatially varying environment. There are allowances for valving gas, and the dropping of ballast. Sun angles are predicted according to the time and latitude/longitude location of the balloon. This code has been checked, calibrated against all recent SPB flights. In particular good correlation was seen for flight 662NT, the 2015 flight from New Zealand to Australia ${ }^{16}$.

Data measured and recorded on actual flights includes ascent rate, altitude, differential pressure and free air temperature. In the absence of a reliable non-invasive technique envelope film temperature is not recorded, although this, coupled with differential pressure, is the key loading data input for the balloon structural analyses. If a BalloonAscent simulation using measured environmental conditions correlates well with actual balloon elevation and differential pressure, then film temperature can reliably be backed out of that simulation.

This latter approach has been used by Cathey to support the derivation of typical representative load cases for this current exercise. Data from flight 662NT forms the basis of this work. Although full documentation is available for the 2016 flight 669NT, also launched from New Zealand, this has not been used as this data showed evidence of gas leakage which introduced a degree of uncertainty into the simulation.

A plot of average differential pressure for flight 662NT is shown in figure 16. Rather than extract the absolute maximum and minimum pressure cases the aim was to extract a 4 day 'typical' sequence of 'average' hot, cold and standard days. Based upon BalloonAscent simulations a linear relationship was established by Cathey that relates differential pressure (DP) to average bulk film temperature. Local nuances were added by introducing temperature offsets to capture differences between the cases for increasing temperature and pressure during the transition from night to day and the reverse for the end of the day. The temperature profile also exhibits a faster ramp up in the morning relative to the increase in DP and a slower cool down in the evening.

The chosen 4 day typical loading module is shown in figure 17. 
Super Pressure Flight 662NT - Average Differential Pressure

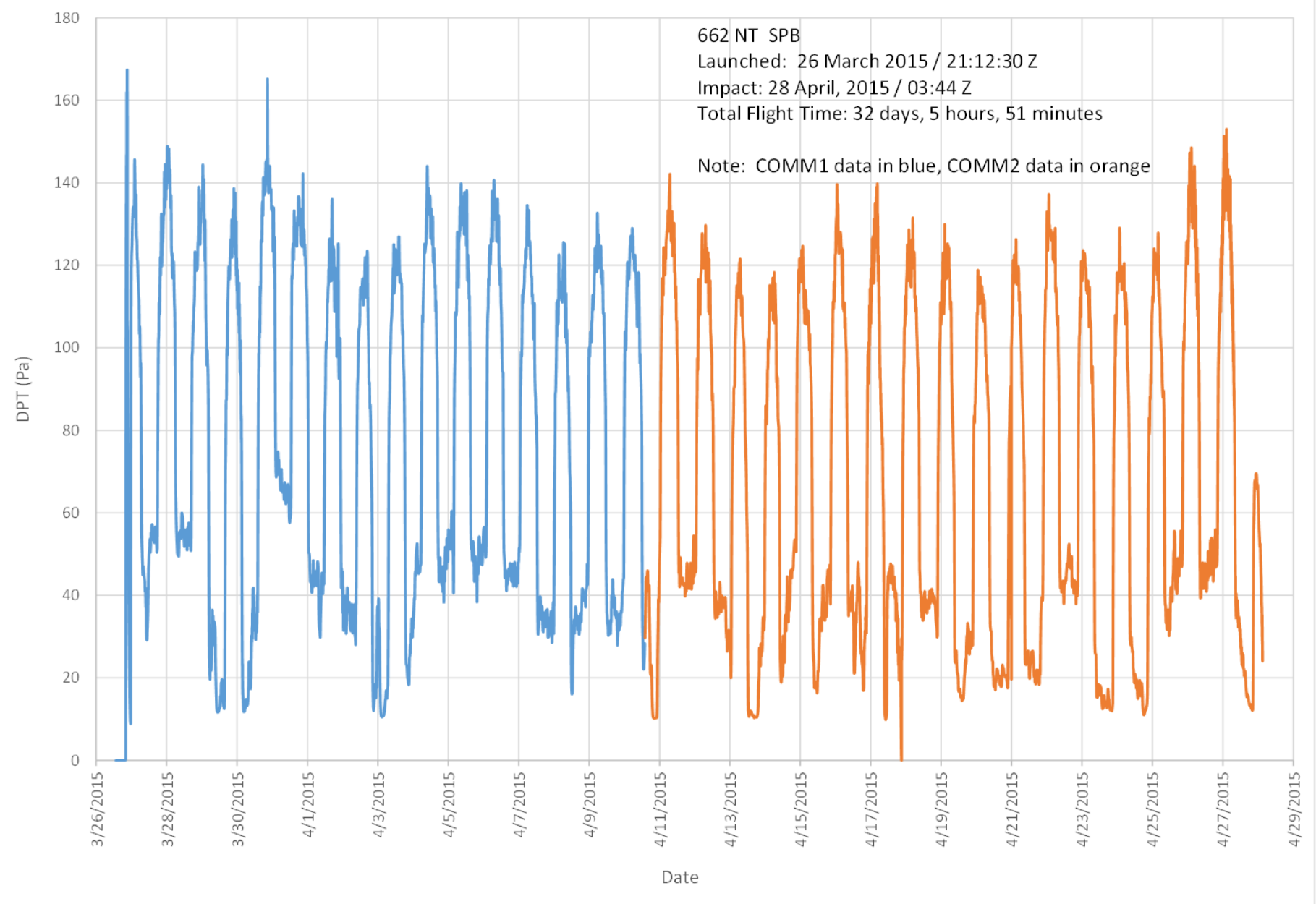

Figure 16. Fight 662NT Instrumentation - Differential Pressure Measurements

\section{Single Gore Model and Time Increment Studies}

For reasons of reduced computational effort, a simplified single-gore balloon model has been used for the development of the incremental analysis procedure and subsequent test and full flight simulations. The model is shown in figure 18, together with a more detailed five gore representation. The main differences with this reduced fidelity single gore model are a smaller number of elements, no separate cap idealisation and lack of cap end castellation. The greatest improvement in computational efficiency of this model comes with the elimination of the separate cap and the associated contact modelling between it and the main gore. The cap is simply represented by an increase in main gore film thickness over its extent. Although there is good film stress and strain correlation between the coarse and refined models, a localised stress concentration develops adjacent to the step in film thickness for the former. This is clearly a function of the model limitation rather than reality and as such is ignored when assessing results. 


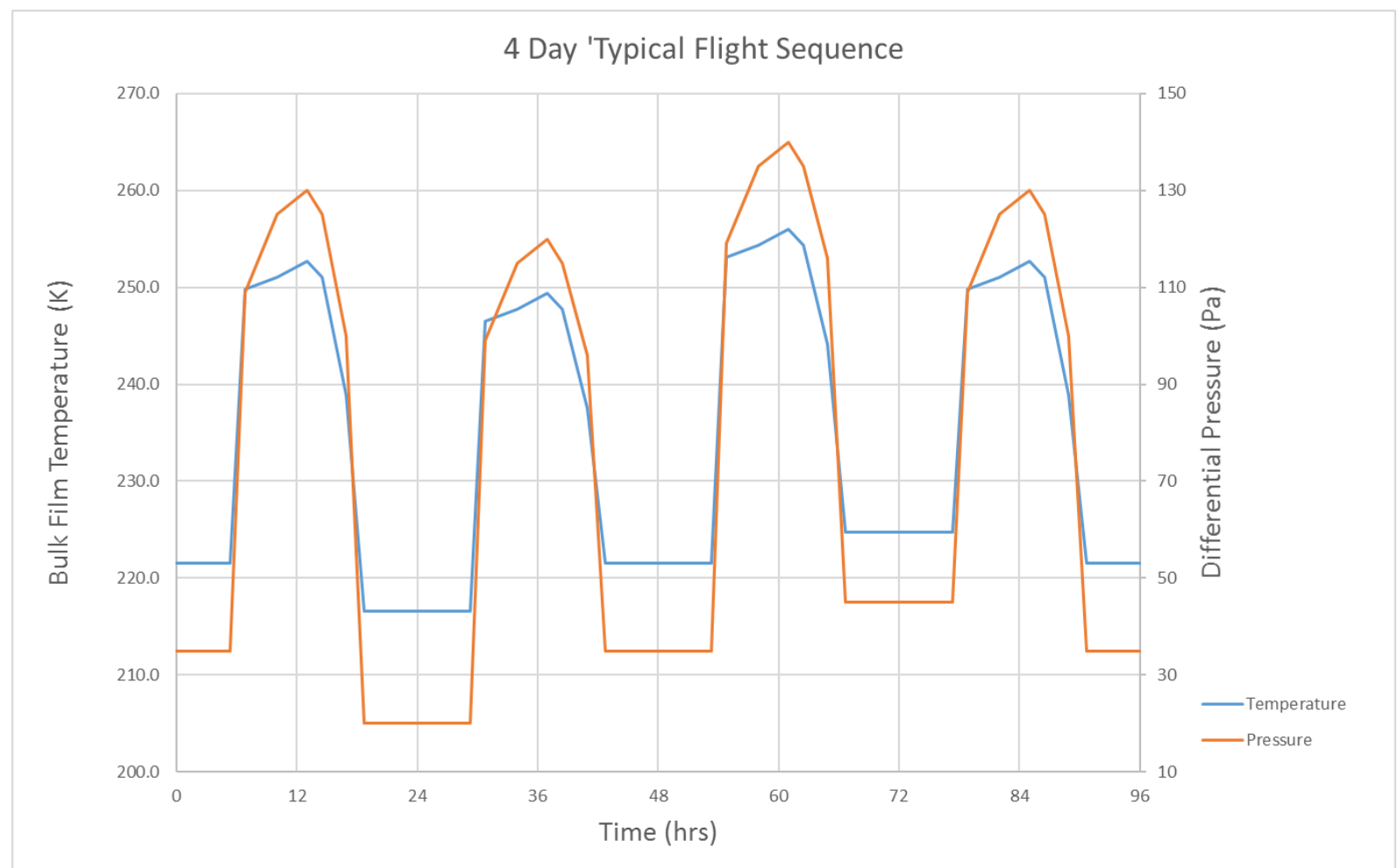

Figure 17. 'Typical' 4 Day Loading Sequence

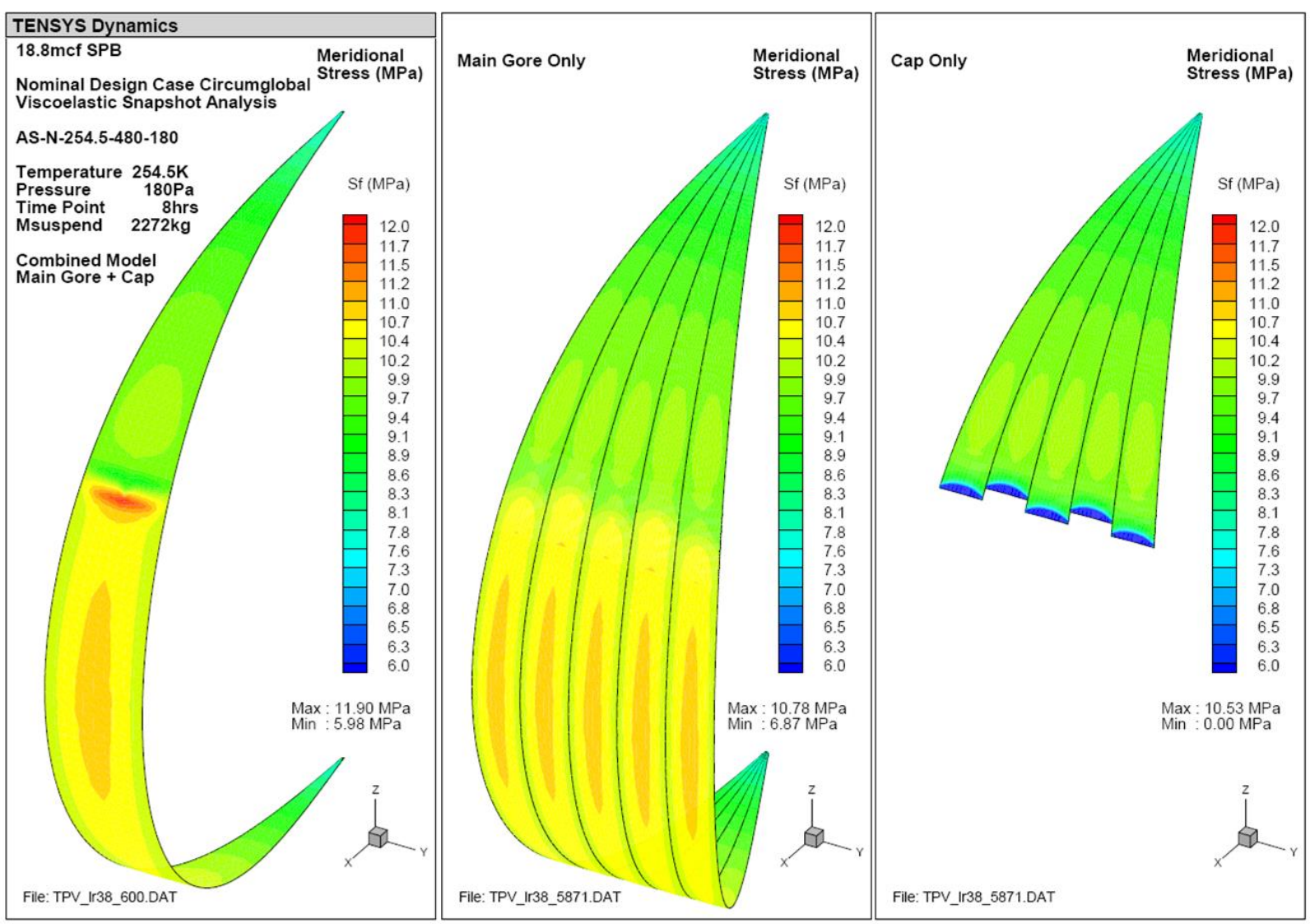

Figure 18. Simplified Single Gore Model and Five Gore Refined Model with Separate Cap

American Institute of Aeronautics and Astronautics 


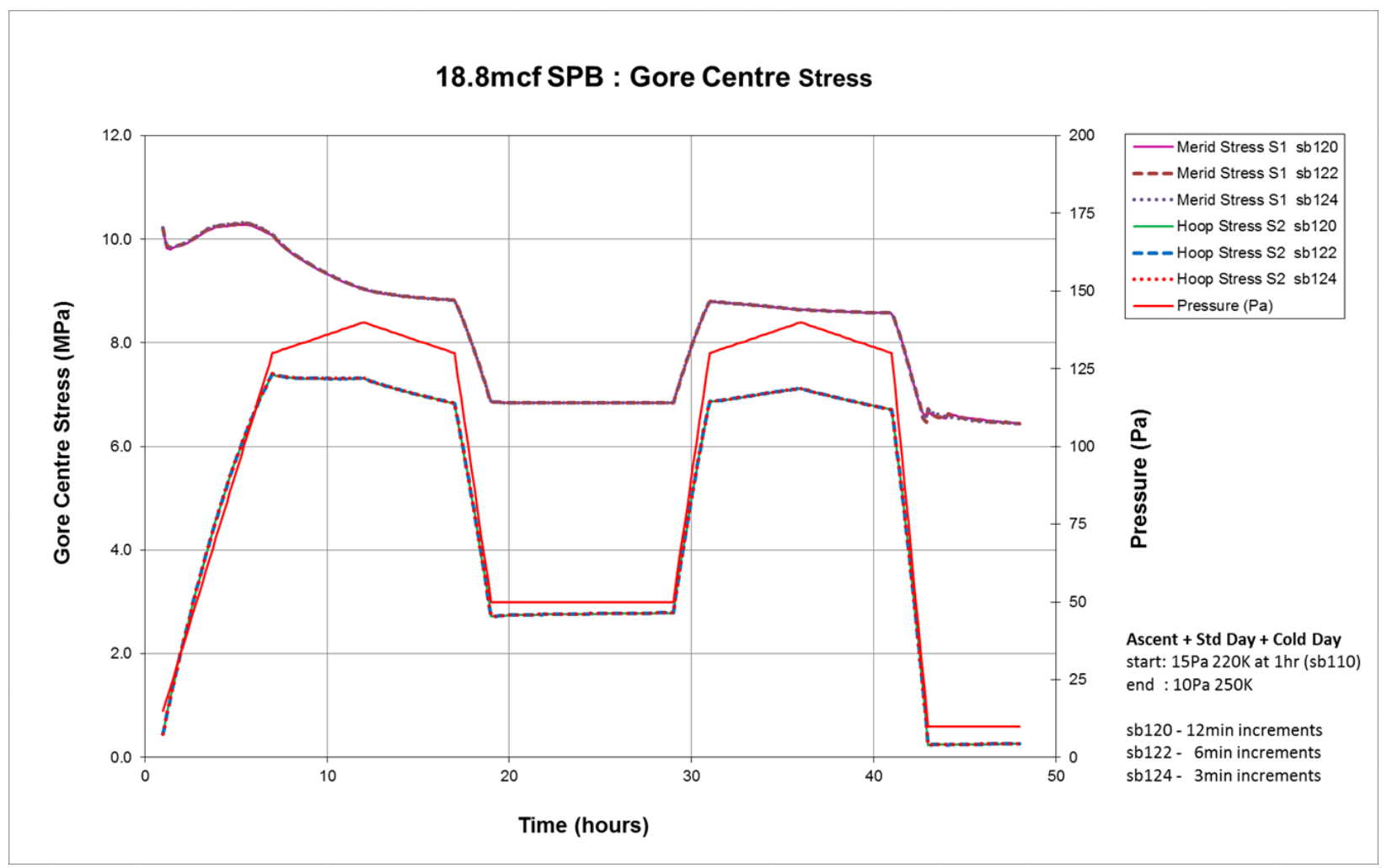

Figure 19. Ascent + Standard Day + Cold Day : Stress Comparison

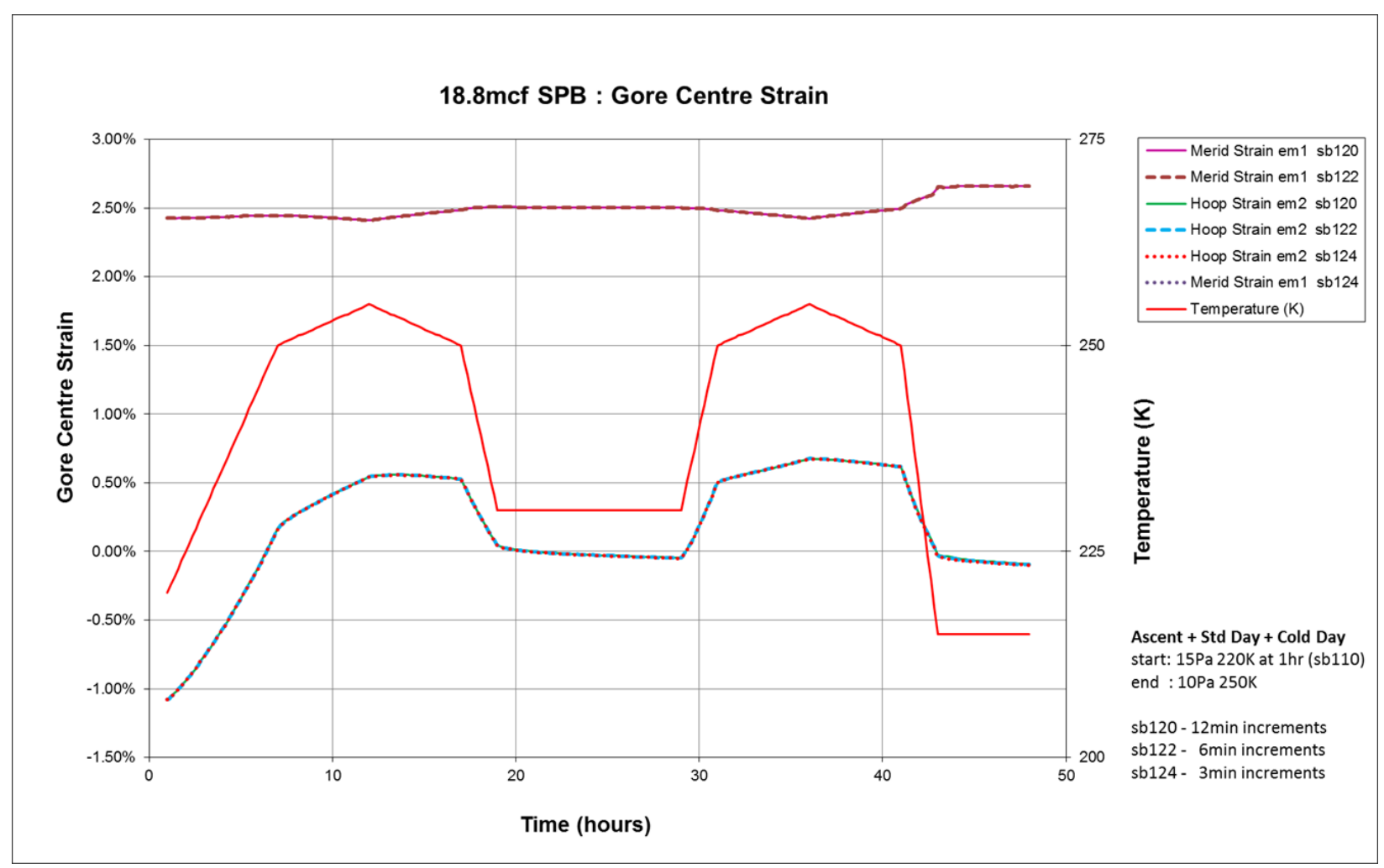

Figure 20. Ascent + Standard Day + Cold Day : Strain Comparison

American Institute of Aeronautics and Astronautics 
Numerical studies using the single gore model were used to ascertain the influence of the time step between successive solutions on the results obtained. These early studies were based upon a simplified 2-day loading sequence that represented ascent, a typical day and a cold day.

Gore centre stresses and strains are plotted on figures 19 and 20 for successive equilibrium analyses undertaken at 3, 6 and $12 \mathrm{~min}$ increments. The results are essentially identical. Previous studies had shown little difference between analyses at 0.3 and 3.0 minute increments. For the reference analyses undertaken here it was decided to adopt a 10minute increment as standard.

For a full 100 day simulation, this implies in excess of 14,000 incremental analyses. The batch processing controls allow for local adjustment of time increments to cover rapidly changing situations such as ascent.

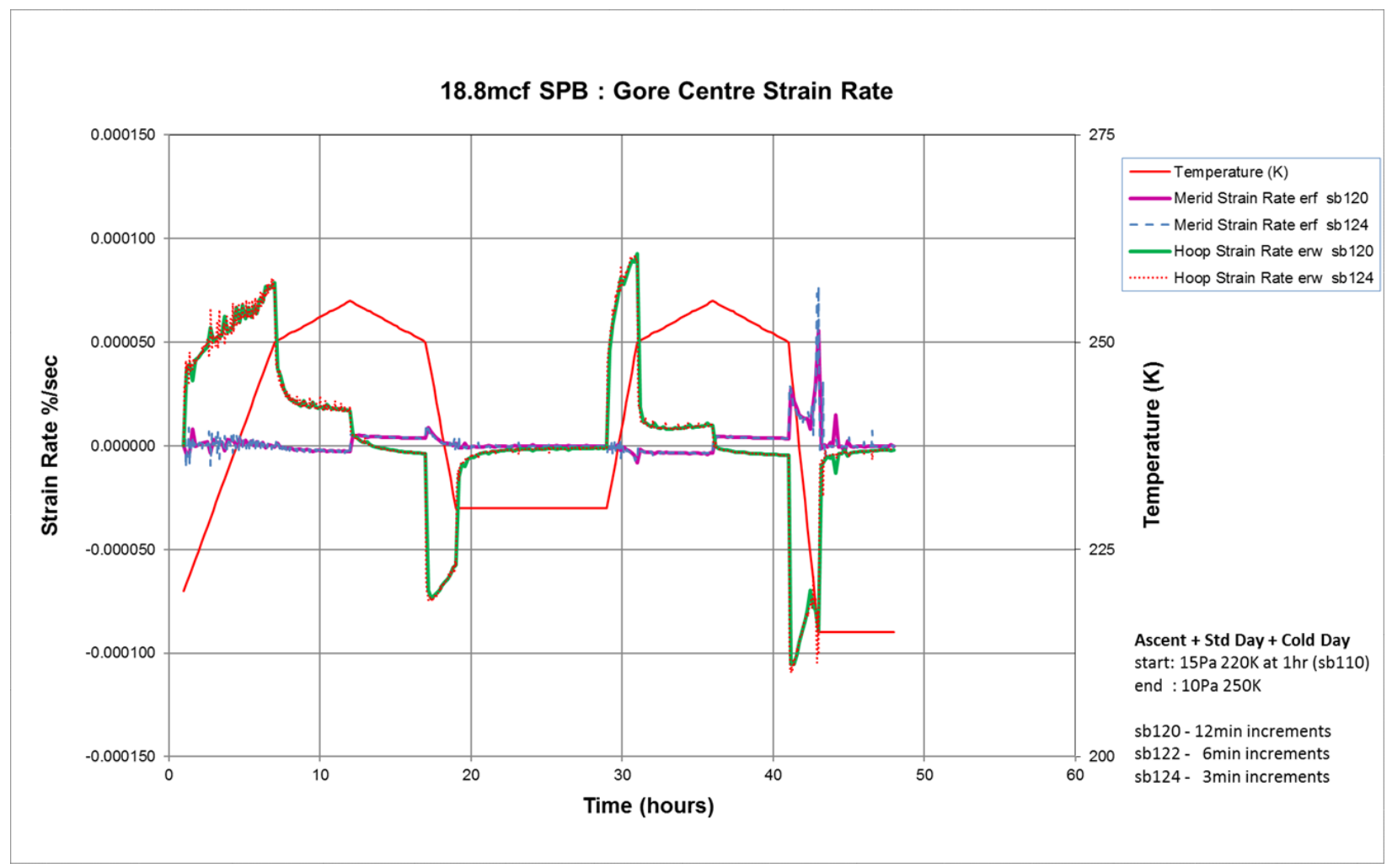

Figure 21. Ascent + Standard Day + Cold Day : Strain Rate Comparison

The same 2-day loading sequence was used to investigate the film rates of strain during these limiting conditions (Fig. 21 ). The gore centre strain rate was below $0.0001 \% / \mathrm{sec}$ throughout. This information informed the strain rates used in the Caltech tests underlying their model coefficients and yield locus determination.

Figure 22 shows the gore centre stresses and strains over a 60 day flight modelled as a succession of the typical four day pressure and temperature loading sequence. The hoop stresses remain essentially unchanged over the flight whilst the meridional stresses reduce by approximately $12 \%$. The hoop mechanical strains increase by approximately $0.3 \%$ whilst those in the meridional direction are effectively unchanged. 

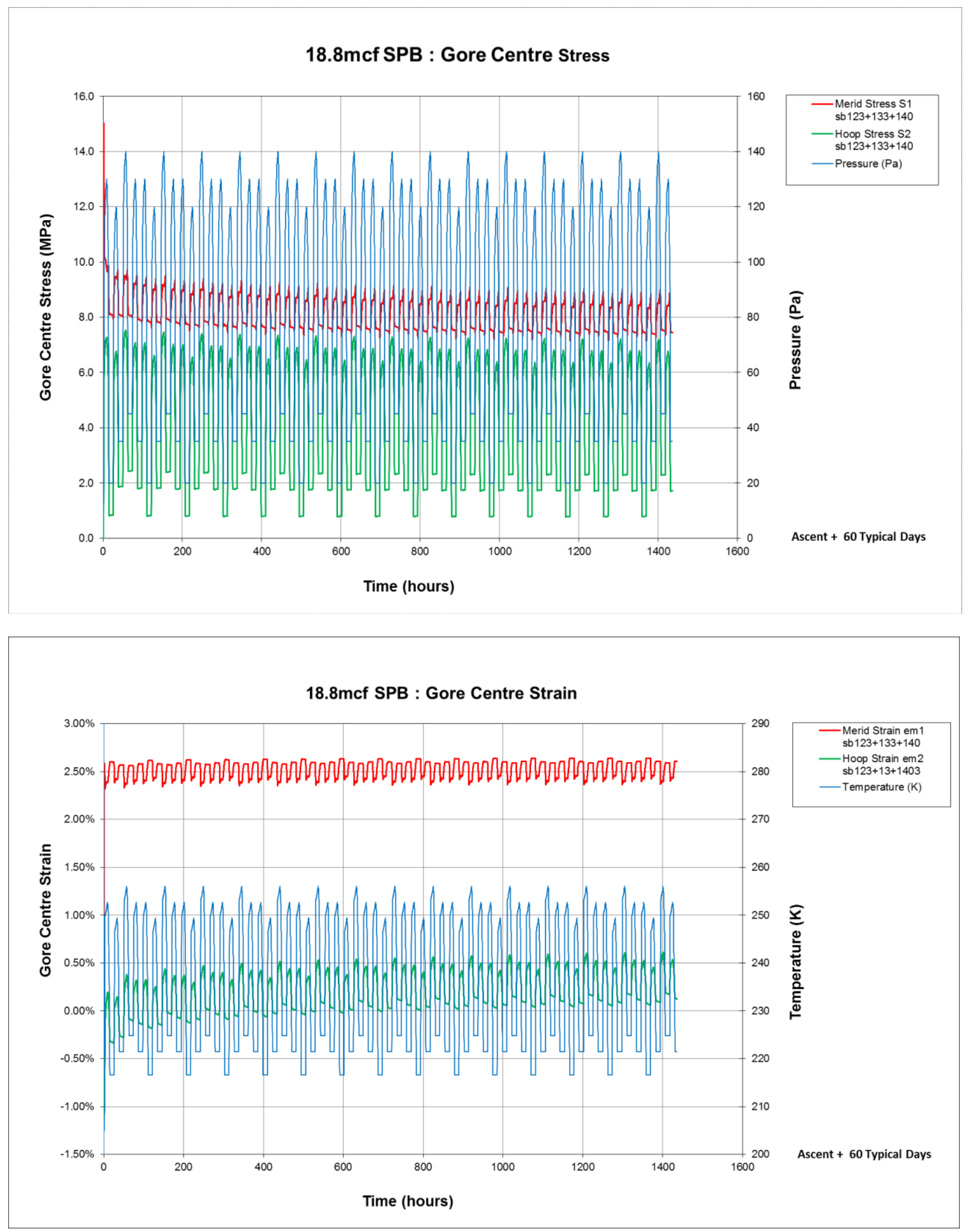

Figure 22. 60 Day Simulation - Gore Centre Stress and Strain 


\section{Comparison with 2 Gore Fine Mesh Model}

Although the analysis development has been based upon a coarser model, it is still intended to implement the same capability with a refined model including a separate castellated cap. This will provide validation of the coarser model but also be the platform for localised studies of the impact of particular details on the balloon structural performance.

Historically these fine mesh studies have been undertaken using a 5-gore model. Given the computational overhead with these studies a 2-gore version of that model has been implemented that retains all the necessary structural variety. The caps are modelled as separate elements with contact active between them and the main gore film. By adopting axes of symmetry that lie though the centre of each gore the effect of cap drop castellation is captured correctly (Fig. 23).

As a guide to the relative computation requirements, simulating 24 hours with the coarse model takes approximately 5 hours, whilst the same with the 2-gore refined model requires 43 hours.

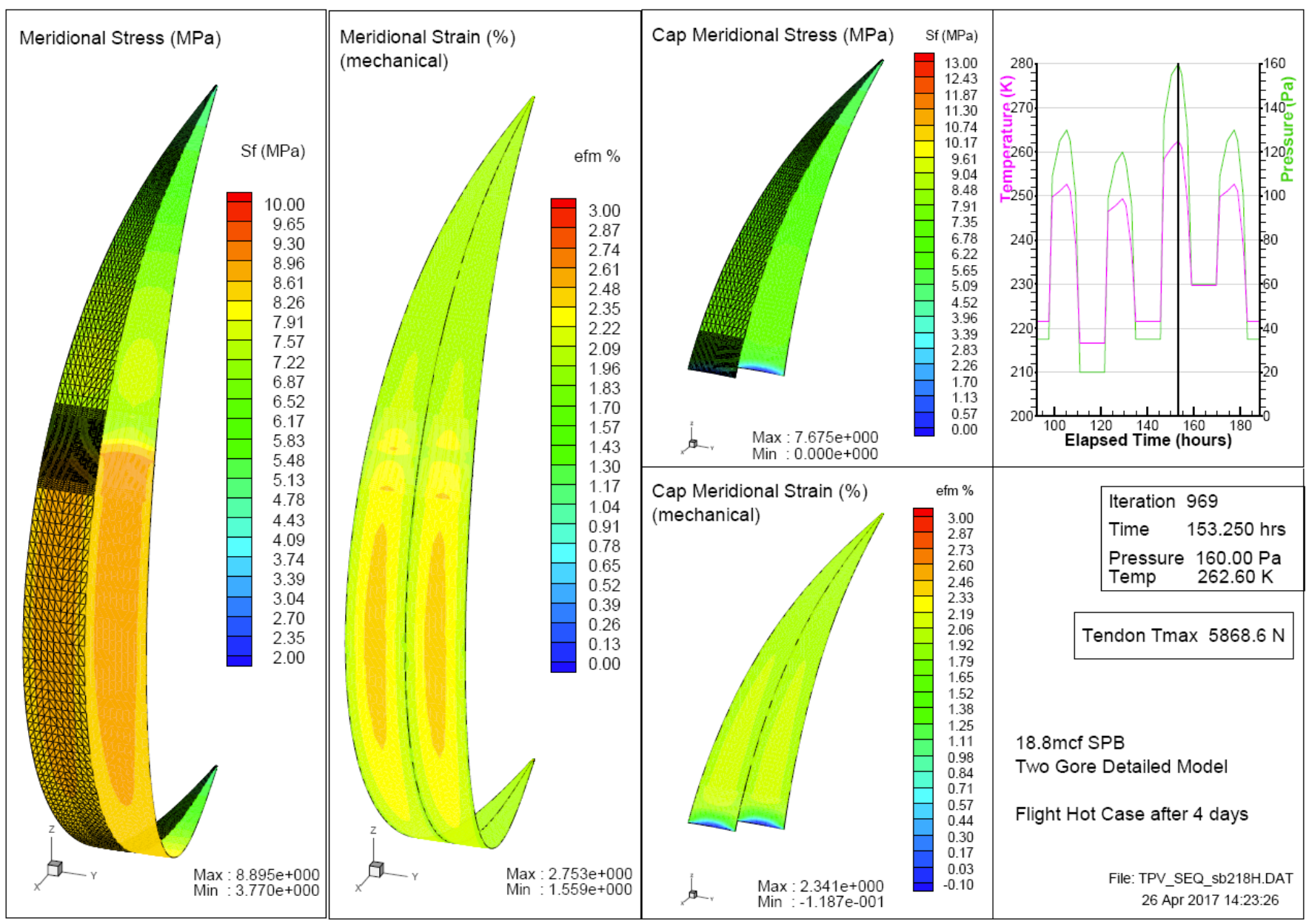

Figure 23. Two Gore Refined Model

First results suggest that the single gore model overestimates gore centre stresses by approximately $3 \%$ in the meridional direction and approximately 9\% in the hoop direction. The latter seems high, and checks are ongoing with the boundary condition and gore unstressed geometry input data 


\section{E. Flight Maximum Load Cases}

Specific maximum and minimum temperature/pressure load cases have been extracted from the flight 662NT data. These are designated the flight hot and flight cold cases respectively. Their loading profiles are shown in figure 24 and the extended load envelope including both typical and flight cases is shown in figure 25. The current SPB design points are also shown in that plot for comparison purposes.

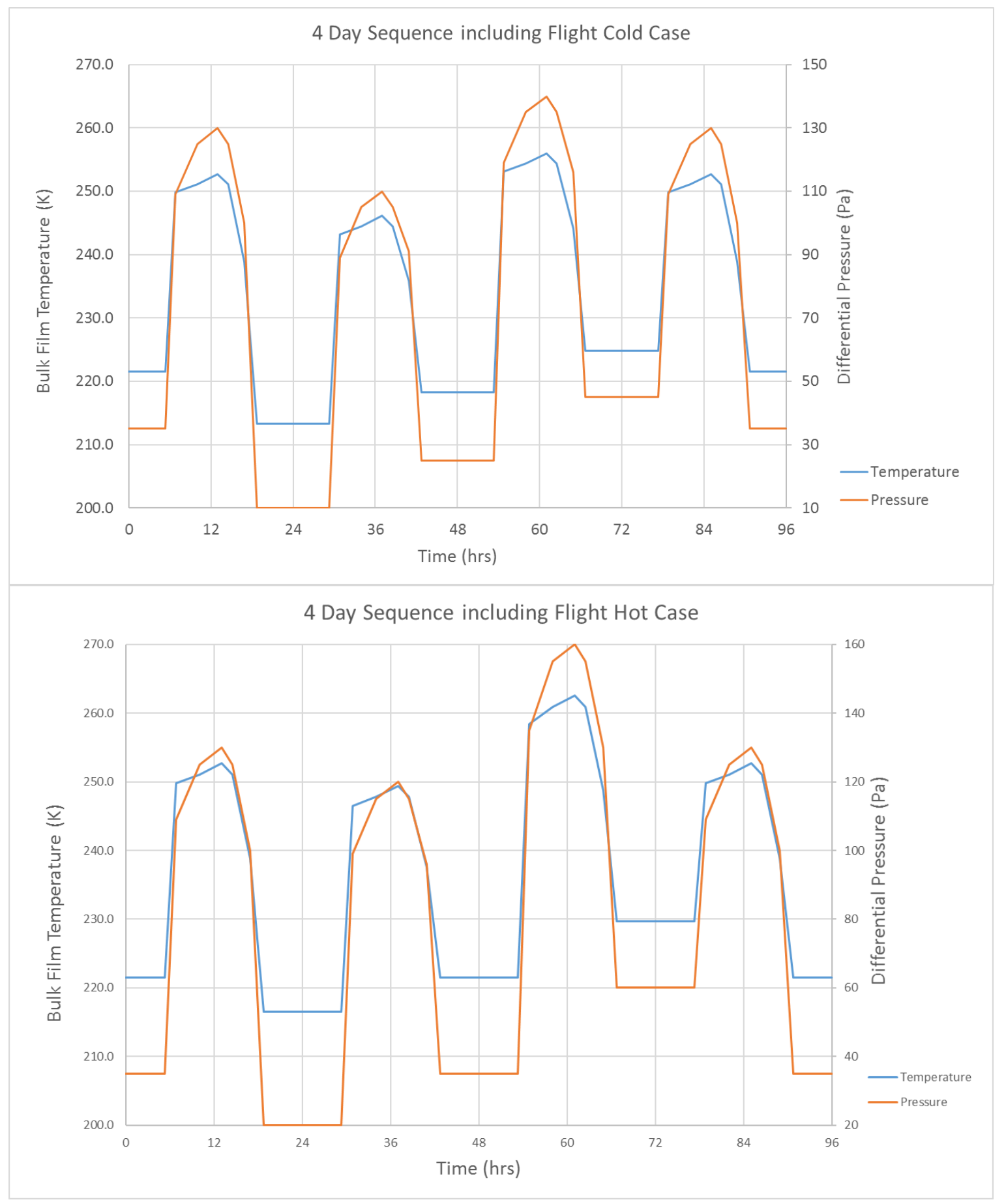

Figure 24. Four Day Sequences including Flight Hot and Cold Cases

American Institute of Aeronautics and Astronautics 


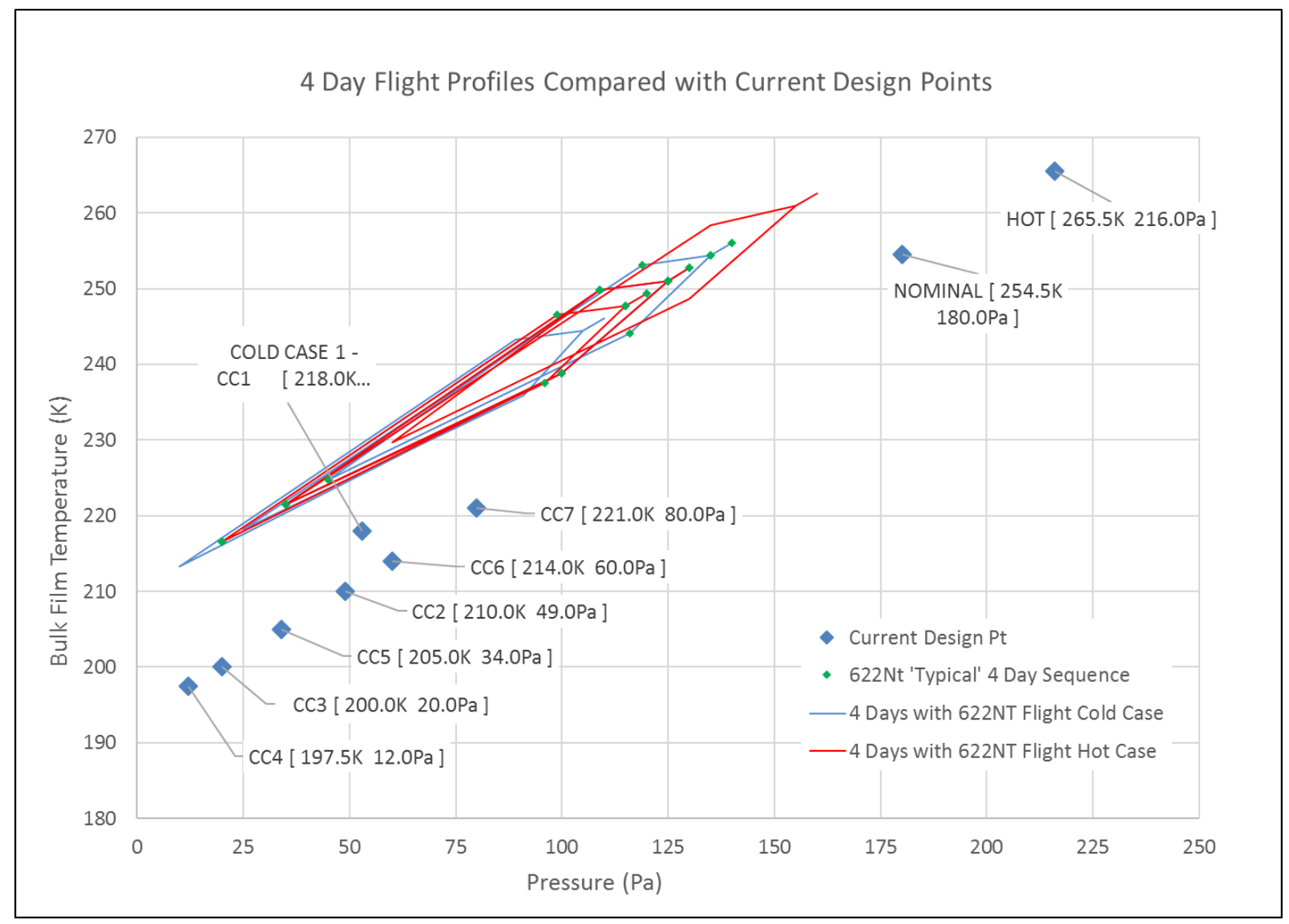

Figure 25. Four Day Flight Profile Loading Envelope

Figures 26 and 27 show the gore centre stresses and strains for the flight hot and cold load cases respectively. The results are shown for those cases applied after 20 and 60 days of the typical flight loading sequence. It can be seen that higher stresses are incurred earlier into the flight.

\section{F. Design Load Cases}

A different procedure is envisaged for the establishment of design load cases associated with this modelling effort. $\mathrm{Bosi}^{18}$ has established a methodology for determining the onset of true yield as a function of temperature and strain rate. The implementation of this criterion is detailed in the next section. Using that capability the current yield factor (defined as yield stress / current stress) is determined for all stages of the simulation. Thus the worst combinations of temperature and pressure can be identified.

It is proposed that the design cases be established by taking these cases and applying an appropriate temperature and/or pressure delta. For example delta $\mathrm{T}=-20 \mathrm{C}$ for the critical cold case and deltaP $=+20 \mathrm{~Pa}$ for the hot case. 


\section{8mcf SPB Flight Hot Case : Gore Centre Stress}
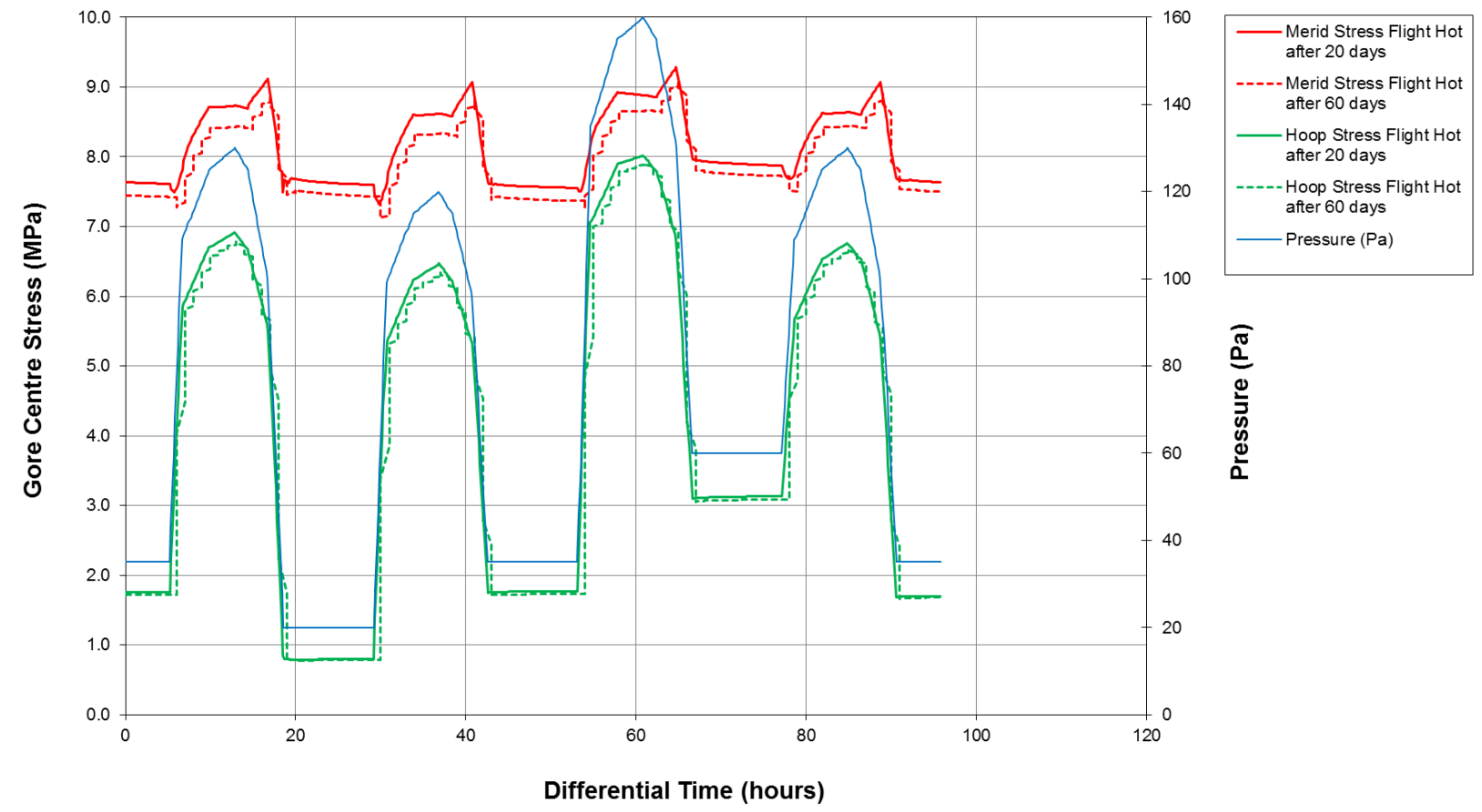

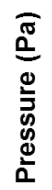

18.8mcf SPB Flight Hot Case : Gore Centre Strain
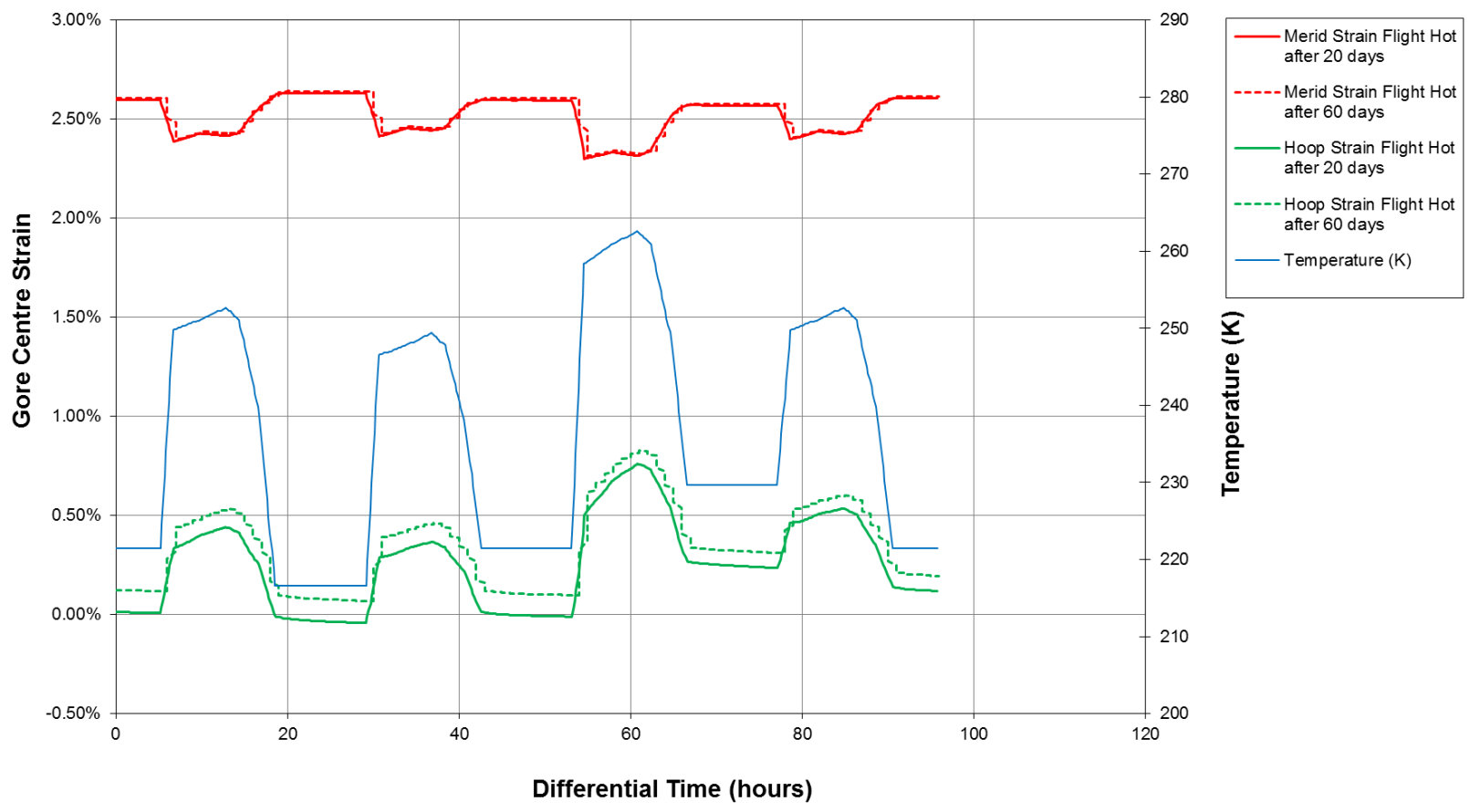

Figure 26. Flight Hot Case Comparison - after 20 and 60 Typical Days 
18.8mcf SPB Flight Cold Case : Gore Centre Stress
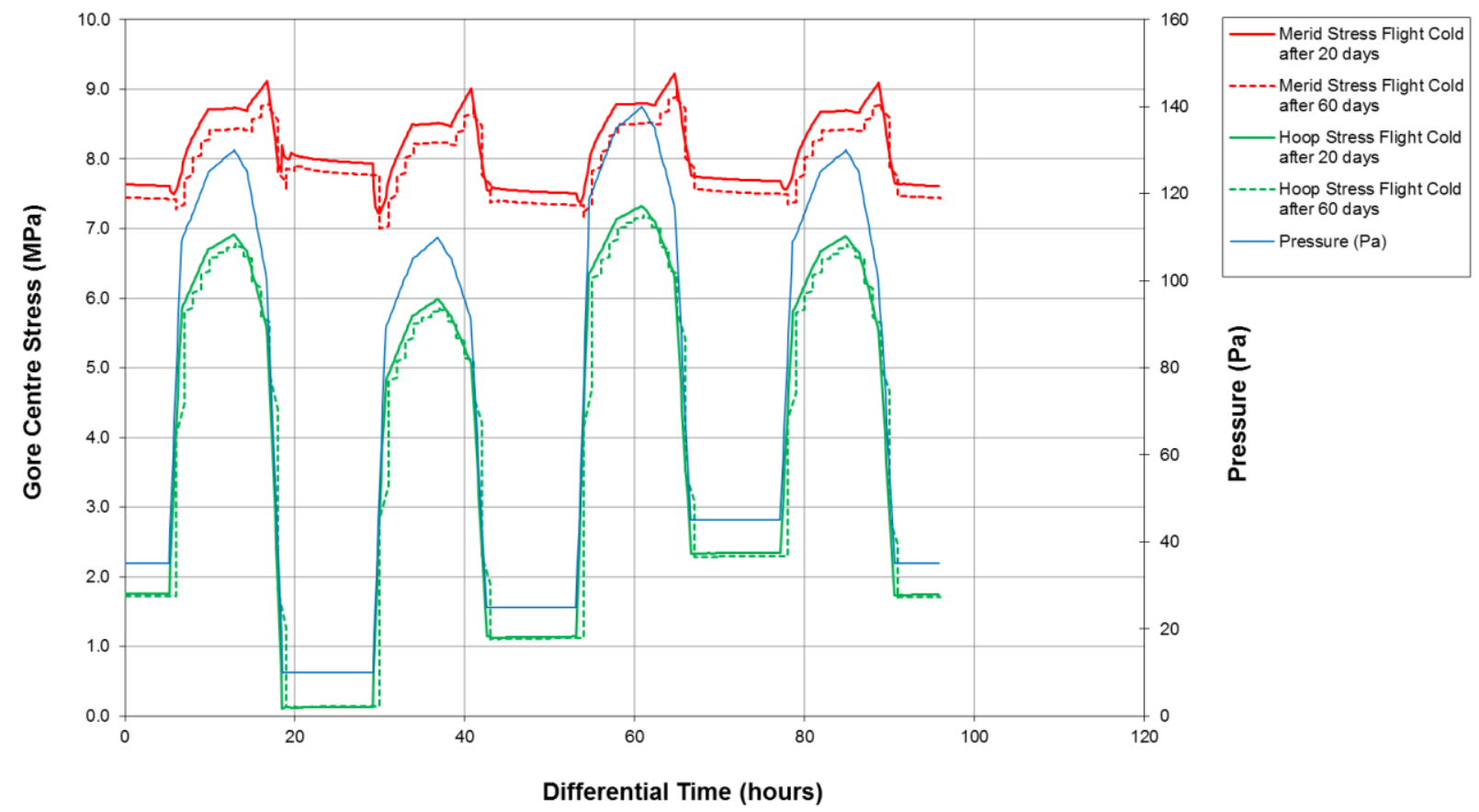

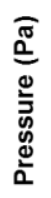

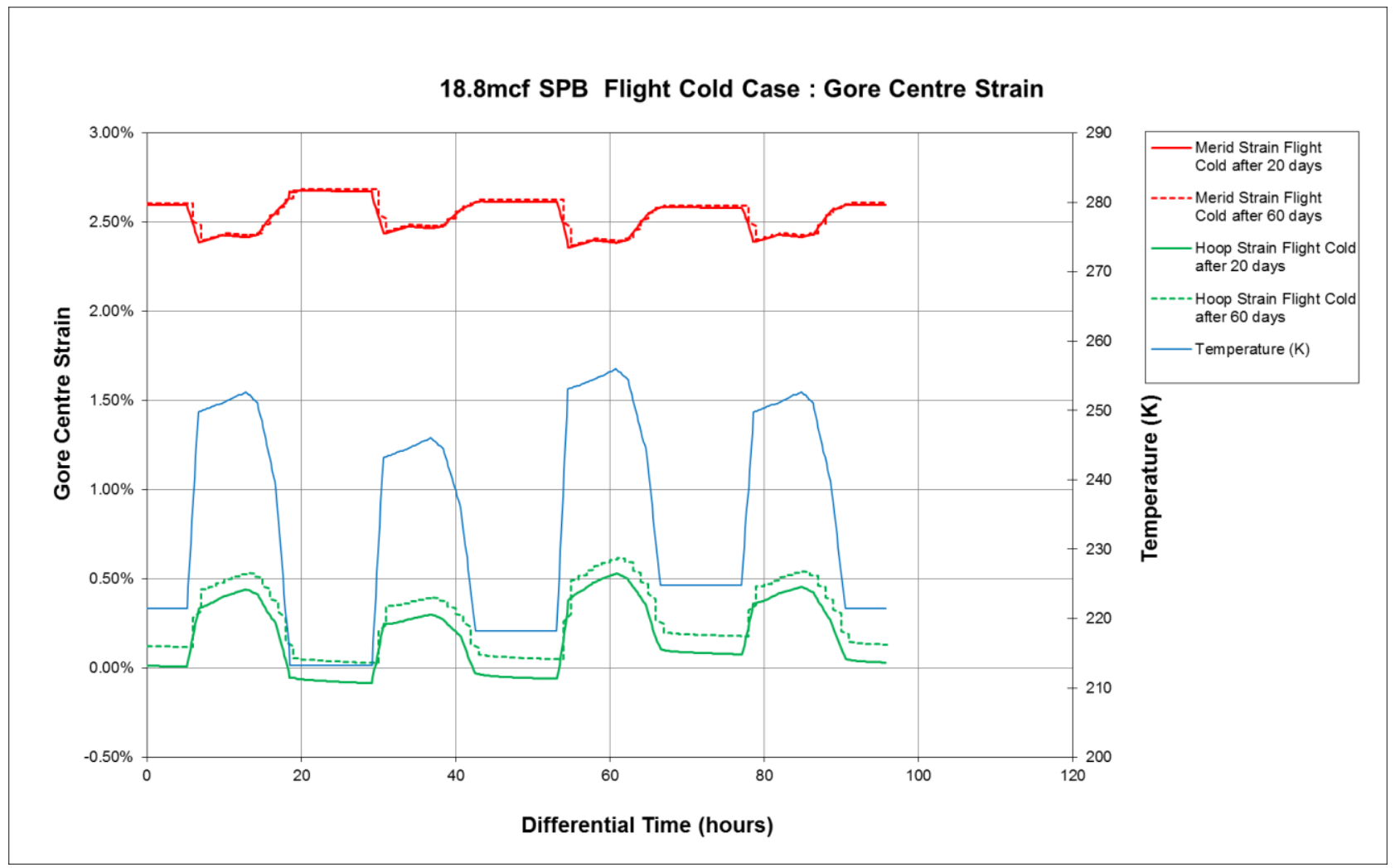

Figure 27. Flight Cold Case Comparison - after 20 and 60 Typical Days

American Institute of Aeronautics and Astronautics 


\section{Design Criteria: Yield Locus}

\section{A. Introduction}

Bosi has proposed yield criteria for the SF420 balloon film based upon yield uniaxial and biaxial tests for true yield at varying strain rates and temperatures. Full details are to be published elsewhere ${ }^{18}$ and the purpose here is a summary of the salient points and details of its initial implementation into the inTENS analysis code.

For uniaxial tests, true yield is defined as the stress that, when unloaded, results in greater than $0.2 \%$ residual strain after 12 hours. The same procedure was applied to bubble tests for equi-biaxial stress. It was also shown that the latter could reliably be assessed by visual inspection of the strain distribution using digital image correlation (DIC).

Plotting a reduced yield stress (yield stress / temperature) against strain rate demonstrated the applicability of strain rate / temperature superposition. Cooperative model fitting was employed to derive a relationship between the temperature reduced yield stress and strain rate.

The yield at any particular MD and TD stress combination and temperature can be calculated for a given strain rate as a function of uniaxial and equi-axial yield stresses. The latter being calculated from temperature and strain rate using the cooperative model mentioned above. The resulting yield stresses may be plotted as loci in 3 dimensions as functions of temperature and strain rate (Fig. 28).

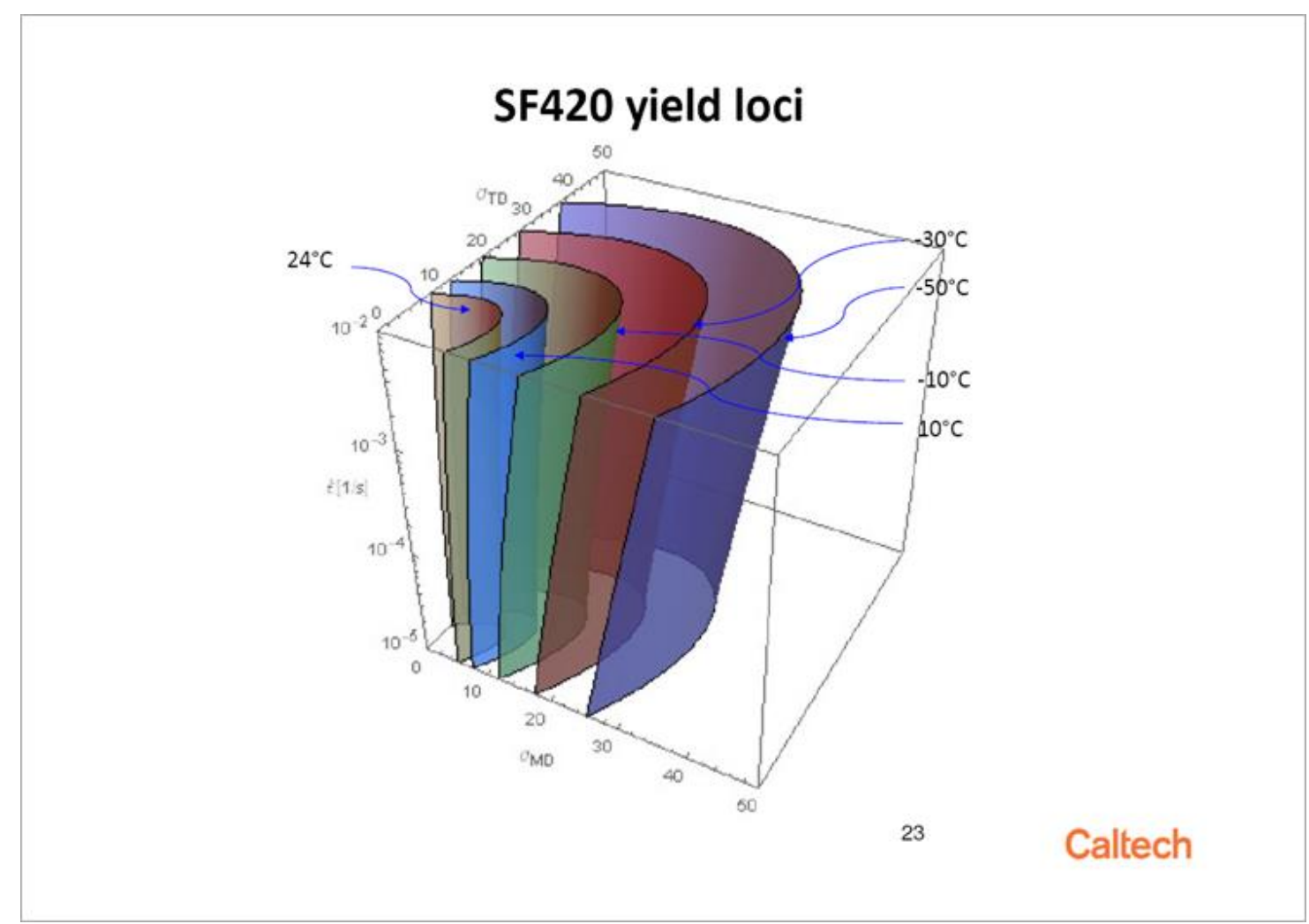

Figure 28. SF420 Yield Loci ( Bosi ) 


\section{B. Yield Locus Practical Implementation}

Application of any such criterion requires investigation as to the suitability of the results in a general analysis context, especially given the relatively small number of test results available. The latter particularly refers to biaxial tests and the complete lack of results for non equi-biaxial conditions.

This work was undertaken by implementing the Caltech formulation and running a series of numerical tests. A reference strain rate of $0.001 \% / \mathrm{sec}$ at $263 \mathrm{~K}$ was used and this is applied in both MD (e1\%) and TD (e2\%) directions in the plot shown in figure 29.

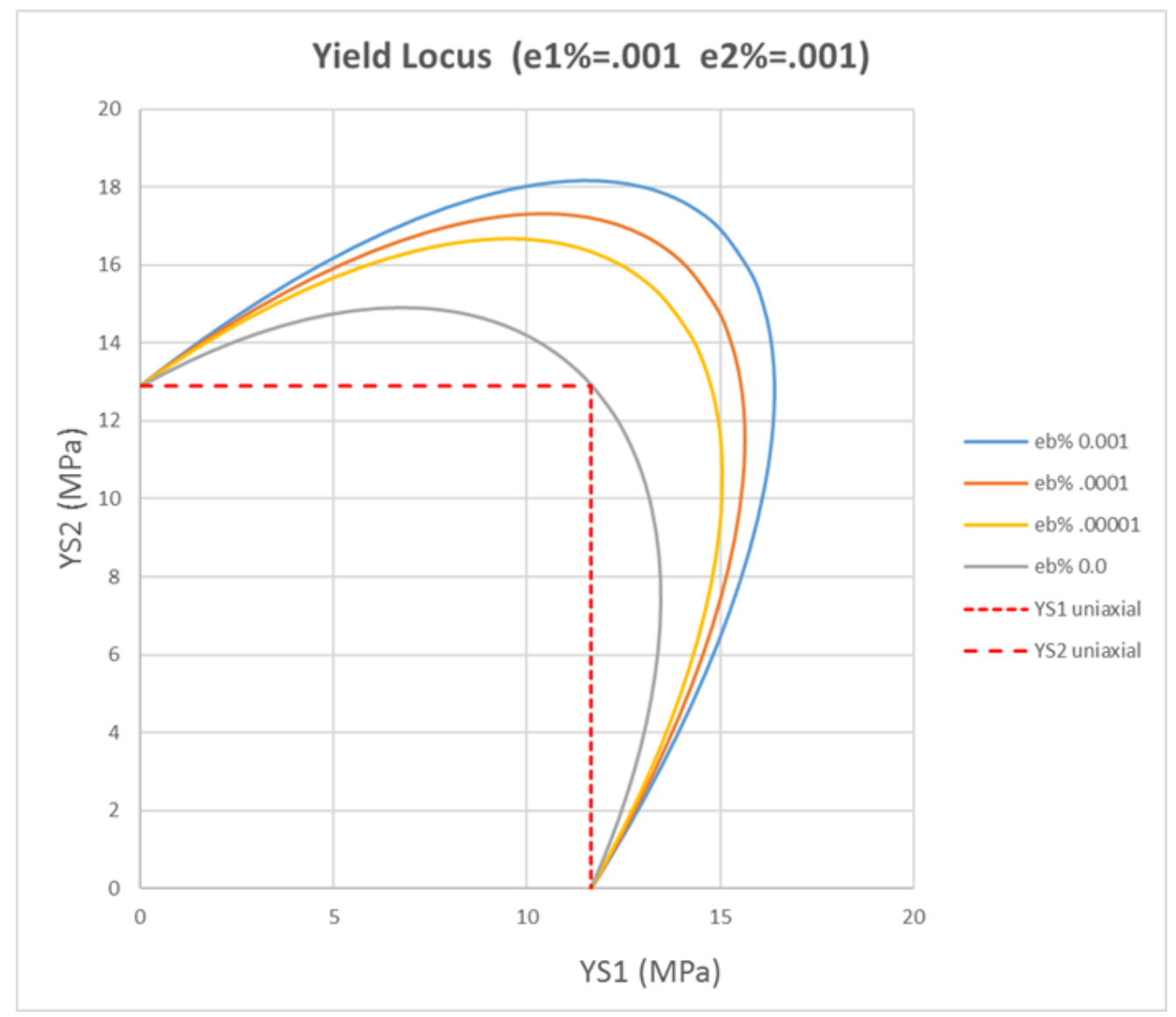

Figure 29. SF420 Yield Loci

The different curves plotted illustrate the effect of changing the assumed equi-biaxial strain rate (eb\%). Blue sets $\mathrm{eb} \%=(\mathrm{e} 1 \%+\mathrm{e} 2 \%) / 2$, in other words the average of the MD and TD rates. Grey represents the extreme with eb\% set to zero, whilst the others represent in between conditions. The understanding of the effect of eb\% on the results is important as it is a variable not immediately available during the analysis. In contrast e $1 \%$ and e $2 \%$ can be determined from the strains at successive time points divided by the intervening time interval.

In figure 30 the TD strain rate is held at the reference value whilst the MD strain rate is progressively reduced. As that rate lowers towards zero the 'distortion' of the yield locus increases ever further away from the uniaxial values. It would not be sensible to utilise these results unless backed up by further testing. 

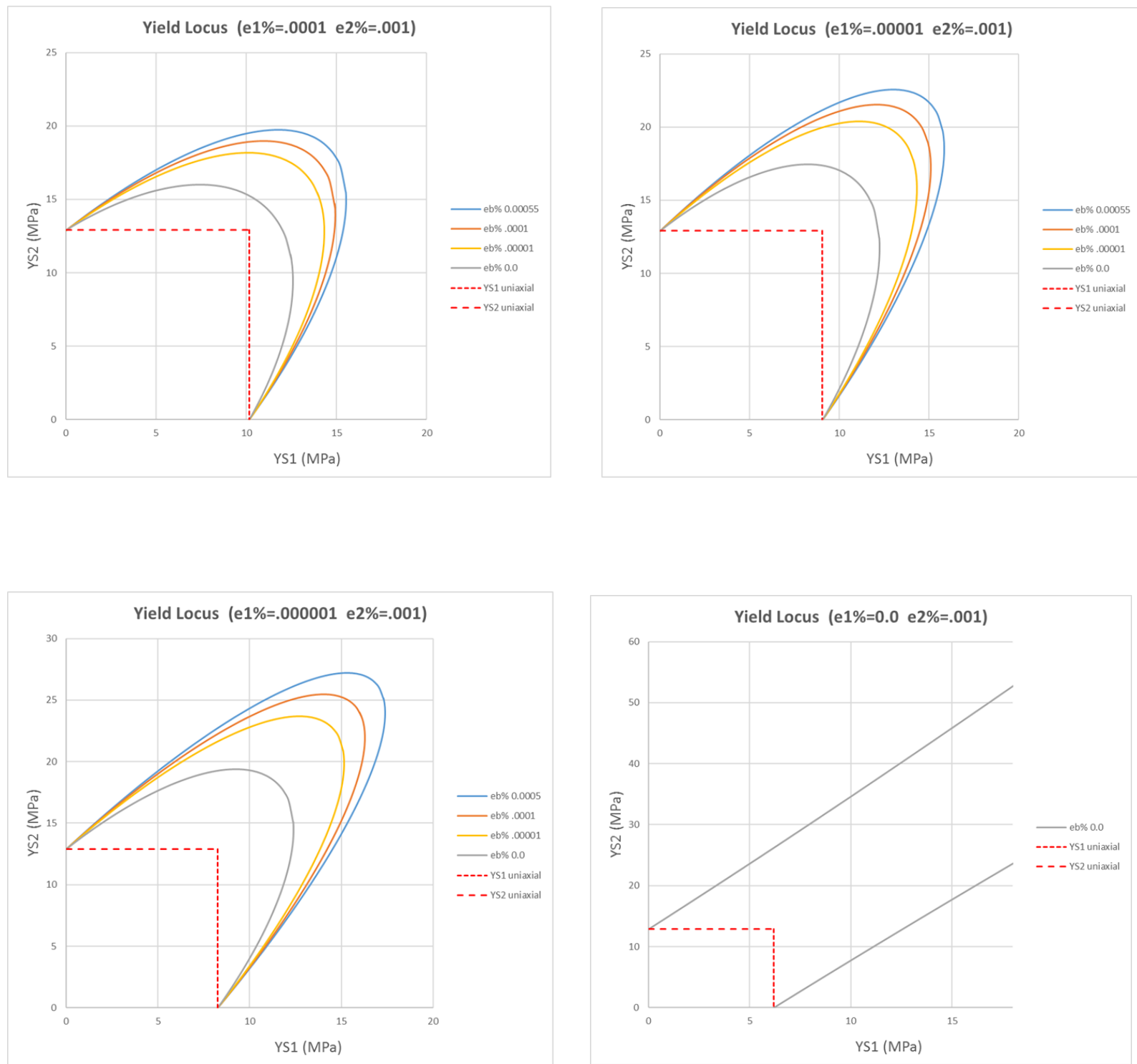

Figure 30. SF420 Yield Loci

The yield loci were developed from tests with minimum strain rates of $0.001 \% / \mathrm{sec}$. Bearing that in mind, and the results above, the Bosi/Caltech locus is currently applied in the following way:

if $\mathrm{MD}$ and TD strain rates (e1\% and e2\%)
both $>0.0001 \%$

otherwise use locus with eb\%=0 to determine yield stresses YS1 and YS2

use the uniaxial values.

This approach is not dissimilar to that adopted for the existing design procedure. In that approach the yield factor is determined by reference to the stress associated with the $2 \%$ offset strain. Both the associated Schapery Rand material model and the load extension curves used for offset strain interpretation were based upon significantly higher strain rates than the balloon sees in practice. These resulted in a material model that was over stiff and higher stress limits. The two were compatible as both model and interpretation were based upon the same loading rates, but not applicable to the current softer model and its slower strain rates. 


\section{Yield Stress Allowables}

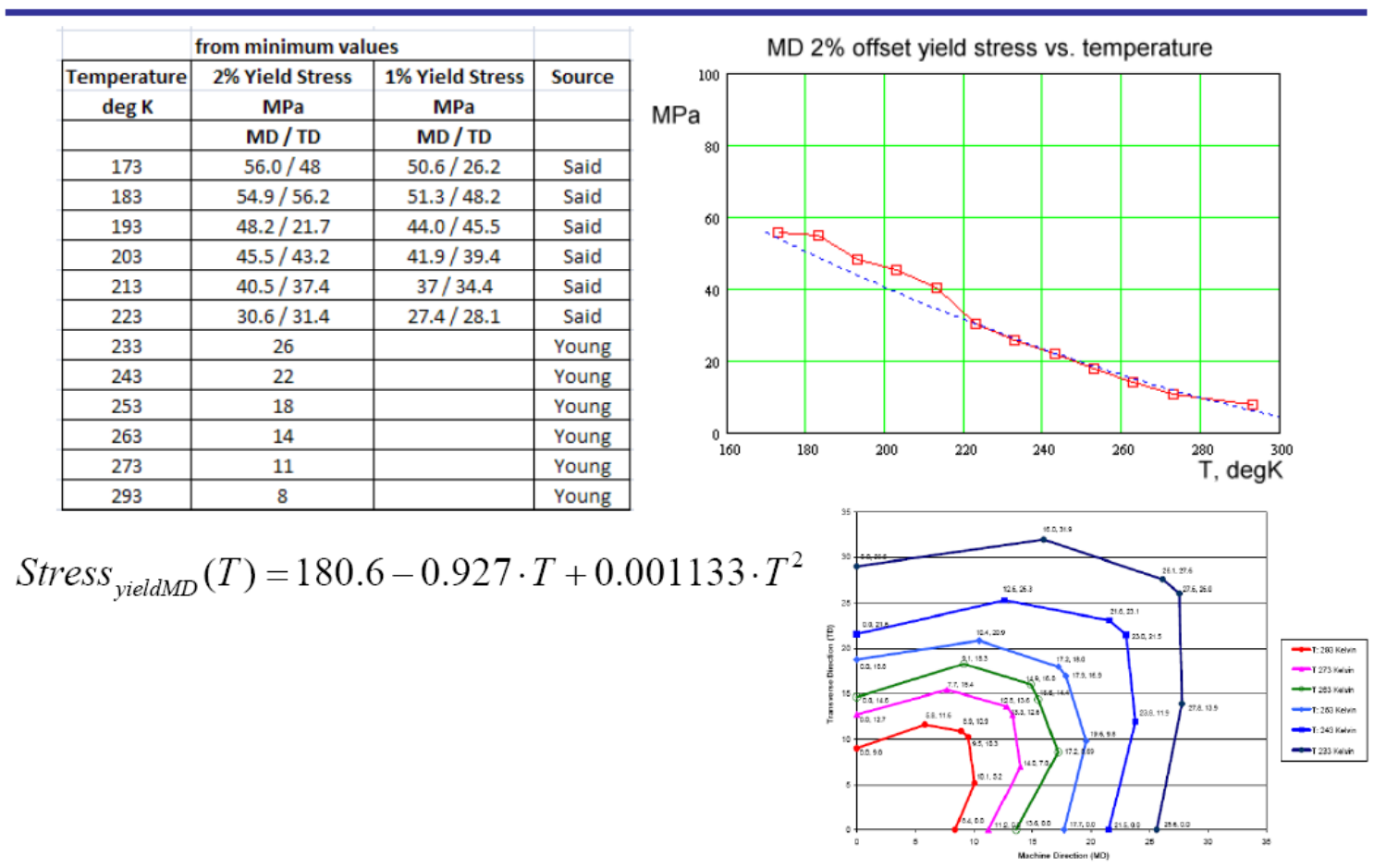

Figure 31. Current Yield Stress Allowables for Schapery Rand Material Model

Figure 31 shows the yield stress allowables used with the Schapery Rand material model. Dependent solely upon temperature, it is the uniaxial values that have been implemented into the design software.

\section{Flight Maximum Cases}

The Bosi/Caltech yield locus has been implemented into inTENS as described above. Figures 32 and 33 show the gore centre yield factors for the flight hot and flight cold load cases applied after 20 typical days. The yield factor is defined as ( yield stress / current stress ).

The most critical yield factors (lowest) for both meridional and hoop directions occur at the point maximum temperature and pressure within the loading sequence. Thus both the typical maximum temperature and pressure combination and the flight maximum are more critical than the flight cold case. In the meridional direction the lowest yield factor was 0.95 for the flight hot case and 1.09 for the flight cold case. Here yield is the onset of permanent deformation, but this is constrained in the meridional direction by the balloon tendons. The lowest yield factors in the hoop direction were 1.26 and 1.55 respectively. These are above the onset of permanent deformation, which is positive as there is no tendon strain arrest equivalent in the hoop direction. 

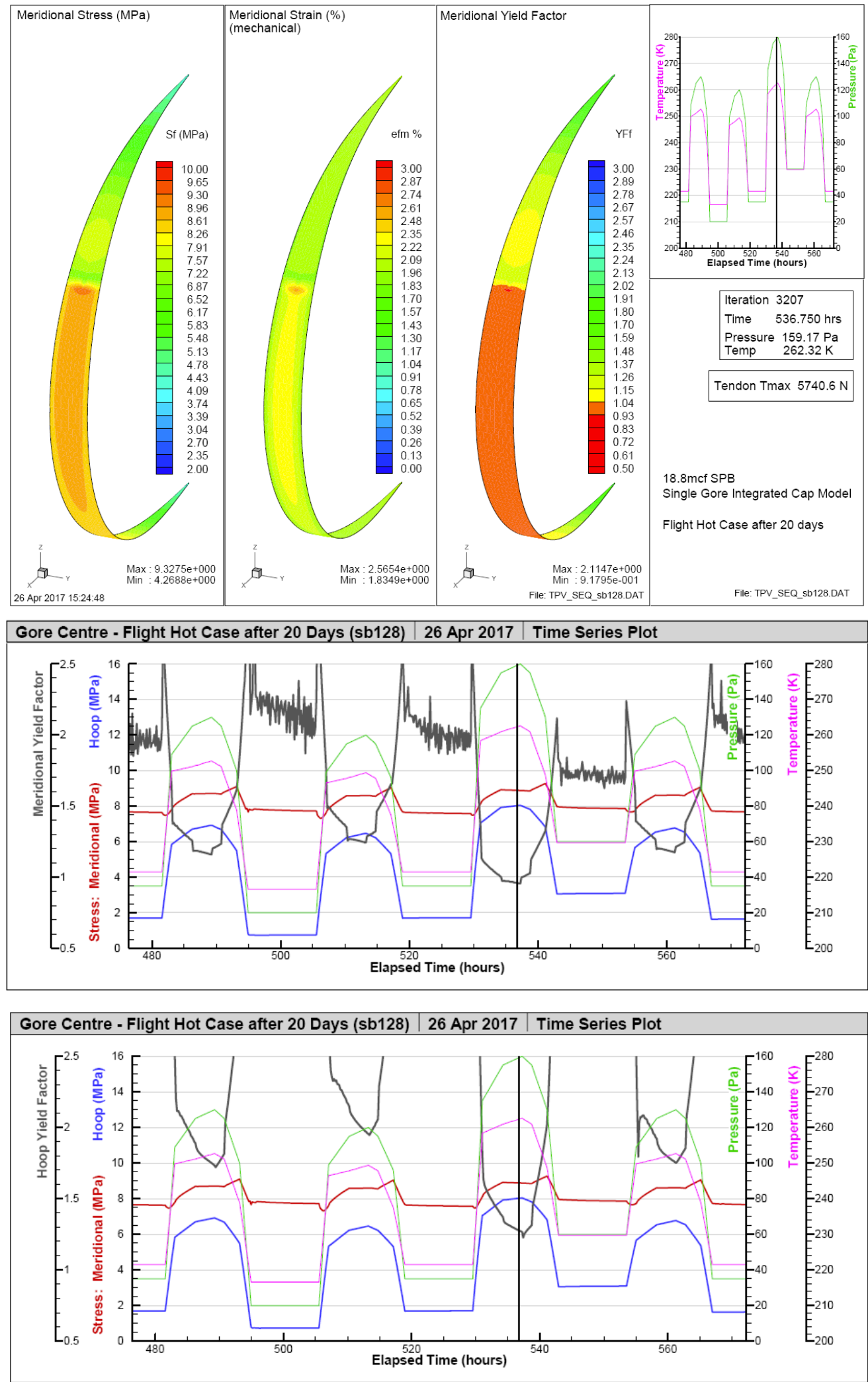

Figure 32. Yield factors - Flight Hot Case after 20 Typical Days 

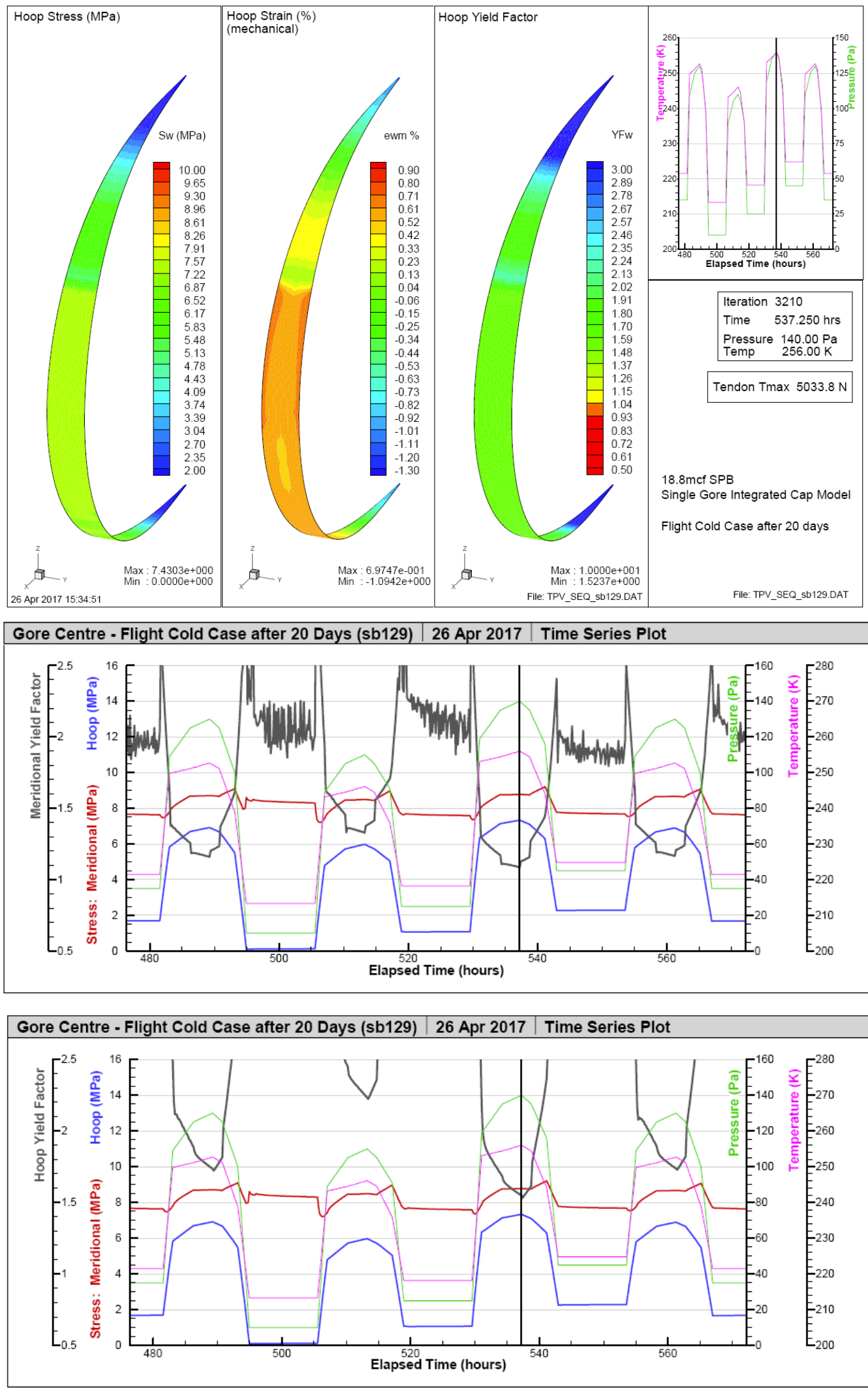

Figure 33. Yield factors - Flight Cold Case after 20 Typical Days 


\section{Summary}

A new large strain thermo-viscoelastic material model for balloon film developed by Pellegrino et $\mathrm{a}^{15}$ has been implemented within the inTENS finite element suite. inTENS is the integrated software package for the form generation, analysis and patterning of lightweight structures developed by Tensys. This package has been utilized over the last 10 years to provide the NASA Super Pressure Balloon Program with stress and stability analysis support. This material model has recently been extended by Bosi ${ }^{18}$ to cover the wider range of operational conditions the material will experience during flight. A longer time domain with slower deformation rates and a wider temperature range has been considered together differing loading conditions including relaxation and cyclic tests

Both snapshot and incremental, time-stepping, approaches have been implemented in inTENS. Convergence issues remain with former, so effort has been focused on the full incremental time-stepping implementation which makes full use of the viscoelastic model without compromise. The key to the approach adopted lies in the fact that all the prior time dependent effects can be encapsulated in a set variables updated at the end of a particular time step. The quantity of data required is the same for any time point independent of whether it lies early or late in a flight sequence. This data need not be stored for each time increment. For example, analyses are typically undertaken at 10 minute time increments whilst the history data is only stored every 12 or 24 hours.

The chosen procedure is to run a time stepping simulation using a repeating typical diurnal temperature and pressure sequence for the full flight duration, say 100 days with regular history data output as above. Specific nonstandard load cases, such as extremes of temperature and pressure, can then be run at any elapsed time in the flight by running that new load case using the associated history data as a start point. If necessary that new analysis can be followed by repeats of the typical loading sequence to assess the long-term effects, if any, of that particular case.

The definition of typical, flight maximum and design loading cases is described, together with the derivation of suitable time steps for the incremental analyses. Both a single gore model, with a simplified cap represented by local film thickening and a two gore refined model with separate cap have been utilised. The former has considerable advantages in terms of computational effort, which is particularly when running 100 day simulations with equilibrium solutions every 10 minutes.

Analyses to date show the combination of high temperature and pressure to be more critical than low temperature cases. Any particular load combination is more critical in the early stages of a flight rather than later. Relaxation of film curvatures due to creep results in lowering stresses over time. The potential influence of this geometry change on the stability of the balloon shape will be studied.

Practical implementation of the yield locus criteria developed by Bosi is described, and sample results presented for flight maximum load cases

\section{Acknowledgments}

The numerical investigations of balloon stress and stability reported here were undertaken by Tensys Dynamics under contract to the Columbia Scientific Balloon Facility, Site Manager Dwayne Orr, for the NASA Balloon Program Office, Program Chief Debora Fairbrother.

This work is an ongoing part of the Super Pressure Balloon team effort, and the authors wish to gratefully acknowledge the cooperation of the NASA, CSBF, PSL/NMSU, Orbital Sciences and Raven Aerostar International personnel involved. Particular thanks are due to Federico Bosi and Sergio Pellegrino of Caltech and Henry Cathey of the University of New Mexico Physical Sciences Laboratory for their contributions to the material characterization and analysis efforts.

\section{References}

\footnotetext{
${ }^{1}$ Wakefield DS, "Numerical Modeling of Pumpkin Balloon Instability", AIAA $5^{\text {th }}$ ATIO and $16^{\text {th }}$ Lighter-than-Air System Technology and Balloon Systems Conferences ,AIAA-2005-7445, 2005

${ }^{2}$ Wakefield DS, "Numerical Investigations of Pumpkin Balloon Instability", AIAA $19^{\text {th }}$ Aerodynamic Decelerator Systems Technology and Balloon Systems Conference, Williamsburg VA,AIAA-2007-2604, 2007
} 
${ }^{3}$ Wakefield DS, "Non-Linear Viscoelastic Analysis and the Design of Super-Pressure Balloons: Stress, Strain and Stability", AIAA $20^{\text {th }}$ Aerodynamic Decelerator Systems Technology, $18^{\text {th }}$ Lighter-Than-Air Systems Technology and Balloon Systems Conferences, Seattle WA ,AIAA-2009-2813, 2009

${ }^{4}$ Wakefield DS \& Bown AC., "Non-Linear Analysis of the NASA Super Pressure Balloons : Some Detailed Investigations of Recent Antarctic Flight Balloons", 11th AIAA ATIO Conference, Virginia Beach, 2011

${ }^{5}$ Wakefield DS, "Engineering Analysis of Tension Structures : Theory and Practice", Engineering Structures ,Vol 21, pp.680690,1999

${ }^{6}$ Wakefield DS, "Structures Cladding and Airships", Membranes 03: Textile Composites and Inflatable Structures, Barcelona, 2003

${ }^{7}$ Rand JL, "A Nonlinear Plasto-Viscoelastic Constitutive Equation for Balloon Films", AIAA 20th Aerodynamic Decelerator Systems Technology, 18th Lighter-Than-Air Systems Technology and Balloon Systems Conferences, AIAA-2009-2812, 2009

${ }^{8}$ Rand JL, "An Improved Constitutive Model for StratoFilm 420", Winzen Engineering Inc. Report, 2008

${ }^{9}$ Pagitz M \& Pellegrino S, “Buckling Pressure of 'Pumpkin’ Balloons”, International Journal of Solids and Structures, 44(21), 6963-6986, 2007

${ }^{10}$ Gerngross T \& Pellegrino S, “Anisotropic Viscoelasticity and Wrinkling of Super Pressure Balloons: Simulation and

Experimental Verification", AIAA 20th Aerodynamic Decelerator Systems Technology, 18th Lighter-Than-Air Systems

Technology and Balloon Systems Conferences, AIAA-2009-2815, 2009

${ }^{11}$ Deng X. \& Pellegrino S, "A technique to predict clefting of lobed super-pressure balloons", 11th AIAA ATIO Conference, 20-22 September, Virginia Beach, AIAA-2011-6830,2011

${ }^{12}$ Kwok K \& Pellegrino S, "Large strain viscoelastic model for balloon film", 11th AIAA ATIO Conference, Virginia Beach, AIAA-2011-6939,2011

${ }^{13}$ Farley RE, "BalloonAscent: 3D Simulation Tool for the Ascent and Float of High-Altitude Balloons", AIAA $5^{\text {th }}$ ATIO and $16^{\text {th }}$ Lighter-than-Air System Technology and Balloon Systems Conferences ,AIAA-2005-7412, 2005

${ }^{14}$ Cathey H \& Pierce D, "Duration Flight of the NASA Super Pressure Balloon”, AIAA 20th Aerodynamic Decelerator Systems Technology, 18th Lighter-Than-Air Systems Technology and Balloon Systems Conferences, AIAA-2009-2808, 2009

${ }^{15} \mathrm{Li}$ J, Kwok K \& Pellegrino S, “Thermoviscoelastic Models for Polyethylene Thin Films”, Mechanics of Time-Dependent Materials, 13(1) 13-43, 2016

${ }^{16}$ Cathey H \& Fairbrother D, Tuttle J, Said M, Farley R., "Qualification of the NASA Super Pressure Balloon", AIAA Balloon Systems Conference Conference, 2015 AIAA Aviation Forum, Dallas

${ }^{17}$ Bosi F \& Pellegrino S, "Nonlinear Thermomechanical Response and Constitutive Modelling of Viscoelastic Polyethylene Membranes", Submitted for Publication

${ }^{18}$ Bosi F \& Pellegrino S, "Molecular Based Temperature and Strain Rate Dependent Yield Criterion for Anisotropic Elastomeric Thin Films", Submitted for Publication

${ }^{19}$ Wakefield DS \& Bown AC., "Non-Linear Analysis of the NASA Super Pressure Balloons : Implementation of a Large Strain Material Model", AIAA Balloon Systems Conference Conference, 2015 AIAA Aviation Forum, Dallas 